\title{
Symmetry, Constitutive Laws of Bounded Smoothly Deformable Media and Neumann Problems
}

\author{
E. Binz
}

No. $108 / 1990$

Dedicated to Werner Greub on the occasion of his $65^{\text {th }}$ birthday.

0. Introduction

1. Configuration and phase space, geometric preliminaries

2. Special one-forms on $E\left(M, \mathbb{R}^{n}\right)$ and $E\left(M, \mathbb{R}^{n}\right) /_{\mathbb{R}^{n}}$

3. The notion of a constitutive law, the Dirichlet integral

4. Force densities associated with constitutive laws

5. Constitutive laws for the boundary

6. The interplay between constitutive laws of boundary and body

7. Simple examples

8. A general decomposition of constitutive laws

9. The rotational symmetry 


\section{Introduction}

The purpose of these notes is to show that symmetry, in particular translational symmetry, is the base on which two notions in the theory of elasticity, associated with deformable bodies in $\mathbb{R}^{n}$, namely constitutive laws and a certain class of force densities are in a one to one correspondence. This correspondence is given by a von Neumann problem converting force densities into constitutive maps which in turn characterize constitutive laws.

As we will see below any constitutive law $\mathrm{F}$ - formulated in our fashion - yields a a stress tensor, as in the usual treatment of elasticity (cf. $[L, L])$. This tensor needs not to be symmetric, however. A criterion for the symmetry is given for constitutive laws invariant and all rotations of $\mathbb{R}^{\mathrm{n}}$.

To describe in short what we mean by a constitutive law, we begin by looking at a moving deformable bounded body in $\mathbb{R}^{n}$. The material should constitute of a deformable medium. The medium forming the boundary may differ from the one

- forming the inside of the body.

Let us first lay out some elements of the geometric setting. We make the geometric assumption that at any time the body is a n-dimensional, compact, connected, oriented and smooth manifold with (oriented) boundary. The boundary needs not necessarily to be connected. During the motion of the body the diffeomorphism type of the manifold with boundary is assumed to be fixed. These assumptions allow us to think of a standard body $M$, which from a geometrical point of view is a manifold diffeomorphic to the one moving and deforming in $\mathbb{R}^{\mathrm{n}}$. Consequently a configuration is a smooth embedding from $M$ into $\mathbb{R}^{\mathbf{n}}$. The configuration space is hence the collection $E\left(M, \mathbb{R}^{n}\right)$ of all smooth embeddings of $M$ into $\mathbb{R}^{n}$. This set equipped with Whitney's $\mathrm{C}^{00}$-topology is a Fréchet manifold (cf.[Bi,Sn,Fi]). A smooth motion of the body in $\mathbb{R}^{\mathbf{n}}$ therefore is described by a smooth curve in $E\left(M, \mathbb{R}^{n}\right)$. The calculus on Fréchet manifolds used in the sequel is the one presented in $[\mathrm{Bi}, \mathrm{Sn}, \mathrm{Fi}]$, which in our setting coincides with the one developed in $[\mathrm{Fr}, \mathrm{Kr}]$.

The physical qualities of the deforming medium enter certainly the work $F(J)(L)$ needed to deform (infinitesimally) the material at any configuration $J \in E\left(M, \mathbb{R}^{n}\right)$ in any direction $L$. The directions are tangent vectors to $E\left(M, \mathbb{R}^{n}\right)$. Since the ladder space is open in the Fréchet space $C^{\infty}\left(M, \mathbb{R}^{n}\right)$ of all smooth $\mathbb{R}^{n}$-valued functions endowed with the $\mathrm{C}^{\infty}$-topology (cf.[Hi]), a tangent vector is thus nothing else but a - function in $C^{\infty}\left(M, \mathbb{R}^{n}\right)$ and vice versa. 
In the following we take $F$, which is a one-form on $E\left(M, \mathbb{R}^{n}\right)$, as the basic entity of 4 our notion of a constitutive law. Throughout these notes F is assumed to be smooth. We do not discuss the question as to whether $F$ characterizes the physical properties of the material fully or not.

To allow only internal physical properties of the material to enter $\mathrm{F}$, we have to specify the notion of a constitutive law somewhat further. Basic to this specification is the fact that these sorts of constitutive properties should not be affected by the particular location of the body in $\mathbb{R}^{n}$. Thus $F$ has to be invariant under the operation of the translation group $\mathbb{R}^{n}$ of the real vector space $\mathbb{R}^{n}$. Moreover we require $F(J)(L)=0$, for any constant map $L$, and any $J \in E\left(M, \mathbb{R}^{n}\right)$ also.

The forms $\mathrm{F}$, which have these two properties, can be regarded as one forms on $\left\{d J \mid J \in E\left(M, \mathbb{R}^{n}\right)\right\}$, where $d J$ is the differential of $J$. This set of differentials is equipped with the $C^{\infty}$-topology as well and is denoted by $E\left(M, \mathbb{R}^{n}\right) /_{\mathbb{R}^{n}}$. The latter space is a Fréchet manifold, too. It admits a natural metric of an $\mathrm{L}_{2}$-type, which is closely related to the classical Dirichlet integral.

- A smooth one-form on $E\left(M, \mathbb{R}^{n}\right) /_{\mathbb{R}^{n}}$ will be denoted by $F_{\mathbb{R}^{n}}$. Hence we deal with i one-forms of the type $\mathrm{F}=\mathrm{d}^{*} \mathrm{~F}_{\mathbb{R}^{n}}$ which are supposed to describe the work done under any distortion. To handle such a one-form $F$ we assume that $F_{\mathbb{R}^{n}}$ can be represented via an integral kernel with respect to the mentioned metric.

A one form $\mathrm{F}$ being of the form $\mathrm{F}=\mathrm{d}^{*} \mathrm{~F}_{\mathbb{R}^{\mathrm{n}}}$ and admitting an integral kernel is called a constitutive law.

It turns out that any constitutive law $\mathrm{F}$ is determined by some smooth map $\mathfrak{H} \in \mathrm{C}^{\infty}\left(\mathrm{E}\left(\mathrm{M}, \mathbb{R}^{\mathrm{n}}\right) / \mathbb{R}^{\mathrm{n}, \mathrm{C}^{\infty}}\left(\mathrm{M}, \mathbb{R}^{\mathrm{n}}\right)\right)$, called a constitutive map.

Hence in our setting we characterize the medium as far as the internal physical properties are encodable in the function $\mathfrak{H}$. This constitutive function $\mathfrak{H}$ determines at any $\mathrm{d} J \in E\left(M, \mathbb{R}^{\mathrm{n}}\right) / \mathbb{R}^{\mathrm{n}}$ two smooth force densities $\Phi(\mathrm{d} J)$ and $\varphi(\mathrm{d} J)$ linked to $\mathfrak{H}(\mathrm{dJ})$ by the following system of equations:

$$
\Delta(J) \mathfrak{H}(\mathrm{dJ})=\Phi(\mathrm{dJ})
$$

and

$$
\mathrm{d} \mathfrak{H}(\mathrm{dJ})(\mathrm{N})=\varphi(\mathrm{dJ})
$$


Here $\Delta(\mathrm{J})$ is the Laplacian determined by the pull back under $\mathrm{J}$ of a fixed scalar

w product on $\mathbb{R}^{n}$. $N$ is the positively oriented unite normal of $\partial \mathrm{M}$ in $\mathrm{M}$. The integrability condition necessary to solve this von Neumann problem of which the force densities are given and the function $\mathfrak{H}$ is the unknown is equivalent with the requirement that

$$
\mathrm{F}(\mathrm{J})(\mathrm{z})=0 \quad \forall J \in \mathrm{E}\left(\mathrm{M}, \mathbb{R}^{\mathrm{n}}\right) \text { and } \quad \forall \mathrm{z} \in \mathbb{R}^{\mathrm{n}}
$$

The constitutive function $\mathfrak{H}$ determines a stress tensor $\mathrm{T}$ given by

$$
\mathrm{T}(\mathrm{J})(\mathrm{X}, \mathrm{Y}):=\langle\mathrm{d} \mathfrak{H}(J) \mathrm{X}, \mathrm{d} J Y\rangle \quad \forall J \in E(M, N)
$$

$X, Y$ vary among all smooth vector fields on $M$. Vice versa any stress tensor yields a constitutive map via the force densities mentioned above.

Since $F^{\prime}$ is also affected by the material forming the boundary, we treat in an

- analogous way the boundary material and exhibit in analogy to $\mathfrak{H}$ a characteristic constitutive map $\mathfrak{h}$. Thus $\Delta(j) \mathfrak{h}(\mathrm{d} J)$ with $\mathrm{j}:=J \mid \partial M$ and $J \in E\left(M, \mathbb{R}^{n}\right)$ describes the * force density $\tilde{\varphi}(\mathrm{d} J)$ up to a constant force density along $\partial \mathrm{M}$. However $\mathrm{d} \mathfrak{H}(\mathrm{d} J)(\mathrm{N})$ also determines force densities which need not to be of the form $\tilde{\varphi}(\mathrm{d} J)$. This - observation allows us to decode the influence of the whole body on the physical quality of the boundary material.

In section 8 we show that both $\mathfrak{H}$ and $\mathfrak{h}$ are structured in the following sense :

In $\mathfrak{H}$ and in $\mathfrak{h}$ is, generically and naturally encoded the work needed to deform volume, area and shape of the body and the boundary respective. The shape is partly expressed by the unite normal vector field $N(j)$ along the embedding $j$ of the boundary. The procedure to decode these influences mentioned is to use an $\mathrm{L}_{2} \rightarrow$ splitting of $\mathrm{d} \mathfrak{h}(\mathrm{d} J)$.

Finally we discuss the symmetry of the stress tensor, which is based on the action of the rotation group on the configuration space..

\section{Acknowledgements:}

* We are indept to Thomas Ackermann and Günter Schwarz for valuable discussions, criticisms and corrections. 


\section{Configuration and phase space, geometric preliminaries}

Let us think of a material body being deformable moving and deforming in the Euclidean space $\mathbb{R}^{n}$. We make the geometric assumption that at any time the body maintains the shape of a n-dimensional, compact, connected, oriented and smooth manifold with (oriented) boundary. The boundary shall not necessarily be connected. The physical qualities of the medium forming the boundary may differ from the ones forming the inside of the body.

Fundamental for our investigation is the assumption that during the motion of the body the diffeomorphism type of the manifold with boundary is assumed to be fixed.

Hence we can think of a standard material body $M$ of which the underlying manifold is diffeomorphic to the body in $\mathbb{R}^{n}$.

The standard body constitutes of a deformable medium and we use $M$ to denote both, the manifold with boundary and the material body.

: From this situation we read off what we mean by a configuration : A configuration is a smooth embedding

$$
\mathrm{J}: \mathrm{M} \rightarrow \mathbb{R}^{\mathrm{n}}
$$

Hence the space of configurations is $E\left(M, \mathbb{R}^{n}\right)$, the collection of all smooth embeddings of $M$ into $\mathbb{R}^{n}$. Endowed with the $C^{\infty}$-topology the configuration space is a Fréchet manifold ( $\mathrm{cf}$.[Bi, $\mathrm{Sn}, \mathrm{Fi}]$ or [Hi]).

Clearly each $J \in E\left(M, \mathbb{R}^{n}\right)$ induces a smooth embedding

$$
\mathrm{J} \mid \partial \mathrm{M}: \partial \mathrm{M} \rightarrow \mathbb{R}^{\mathrm{n}}
$$

a configuration of the boundary $\partial \mathrm{M}$ of the body. Let us denote the collection of all smooth embeddings of $\partial \mathrm{M}$ into $\mathbb{R}^{\mathrm{n}}$ by $\mathrm{E}\left(\partial \mathrm{M}, \mathbb{R}^{\mathrm{n}}\right)$. The latter space endowed with the $\mathrm{C}^{\infty}$-topology is also a Fréchet manifold.

Next let us determine the phase space. The set $E\left(M, \mathbb{R}^{n}\right)$ is obviously a subset of - $C^{\infty}\left(M, \mathbb{R}^{n}\right)$, the collection of all smooth $\mathbb{R}^{n}-$ valued maps of $M$. We equip it with the $\mathrm{C}^{\infty}$-topology, also. Since $\mathrm{M}$ is compact, $\mathrm{C}^{\infty}\left(\mathrm{M}, \mathbb{R}^{n}\right)$ is a complete metrizable locally

- convex space, a so-called Fréchet space.

The phase space is therefore

$$
\mathrm{TE}\left(\mathrm{M}, \mathbb{R}^{\mathrm{n}}\right)=\mathrm{E}\left(\mathrm{M}, \mathbb{R}^{\mathrm{n}}\right) \times \mathrm{C}^{\infty}\left(\mathrm{M}, \mathbb{R}^{\mathrm{n}}\right) .
$$


- Proceeding for $\partial \mathrm{M}$ as for $\mathrm{M}$ we obtain $\mathrm{E}\left(\partial \mathrm{M}, \mathbb{R}^{\mathrm{n}}\right)$ as an open subset of the Fréchet space $C^{\infty}\left(\partial M, \mathbb{R}^{n}\right)$ (cf.[Hi]). Its tangent bundle is obviously trivial. The phase space for the boundary is hence $E\left(\partial M, \mathbb{R}^{n}\right) \times C^{\infty}\left(\partial M, \mathbb{R}^{n}\right)$.

In the sequel of these notes we write $O_{\partial}$ instead of $\left\{J|\partial M| J \subset C^{\infty}\left(M, \mathbb{R}^{n}\right)\right\}$. The map assigning to any $J \in E\left(M, \mathbb{R}^{n}\right)$ its restriction $J \mid \partial M$ is called $R$.

On the configuration space we have two natural actions namely

$$
t: E\left(M, \mathbb{R}^{n}\right) \times \mathbb{R}^{n} \rightarrow E\left(M, \mathbb{R}^{n}\right)
$$

and

$$
\mathfrak{s}: S O(n) \times E\left(M, \mathbb{R}^{n}\right) \rightarrow E\left(M, \mathbb{R}^{n}\right)
$$

assigning to each $J \in E\left(M, \mathbb{R}^{n}\right)$ and each $z \in \mathbb{R}^{n}$ the embedding $J+z$ and goJ respectively for each $g \in S O(n)$. These actions reflect the translational and the rotational symmetry of $E\left(M, \mathbb{R}^{n}\right)$ respectively.

$t$ and $\mathfrak{s}$ extend obviously to $C^{\infty}\left(M, \mathbb{R}^{n}\right)$. The groups $\mathbb{R}^{n}$ and $S O(n)$ act accordingly on $=\mathrm{E}\left(\partial \mathrm{M}, \mathbb{R}^{\mathrm{n}}\right)$. These actions restrict to $\mathrm{O}_{\partial}$ and obviously both extend also to : $\mathrm{C}^{\infty}\left(\partial \mathrm{M}, \mathbb{R}^{\mathrm{n}}\right)$.

The orbit spaces of the respective actions of the translation group $\mathbb{R}^{\mathrm{n}}$ are denoted by $C^{\infty}\left(M, \mathbb{R}^{n}\right) /_{\mathbb{R}^{n}}, C^{\infty}\left(\partial M, \mathbb{R}^{n}\right) /_{\mathbb{R}^{n}}, E\left(M, \mathbb{R}^{n}\right) / \mathbb{R}^{n}, E\left(\partial M, \mathbb{R}^{n}\right) / \mathbb{R}^{n}$ and $O \partial^{n} \mathbb{R}^{n}$.

The nature of these spaces are easily understood if we introduce for any $\mathrm{L} \in \mathrm{C}^{\infty}\left(\mathrm{M}, \mathbb{R}^{\mathrm{n}}\right)$ the differential $\mathrm{dL}$ which is locally given by the Fréchet derivative. The tangent map TL of $L$ is $(L, d L)$. The respective notion of $l \in C^{\infty}\left(\partial M, \mathbb{R}^{n}\right)$ is introduced accordingly.

Hence the orbit spaces mentioned above are nothing else but spaces of differentials of the elements of those spaces on which $\mathbb{R}^{\mathrm{n}}$ acts.

For our later investigations we observe that $M$ and $\partial M$ inherit via respective embeddings into $\mathbb{R}^{\mathrm{n}}$ some basic geometric structures described just below. Let us fix - a scalar product $<,>$ and a normed determinant function $\underline{\Delta}$ (cf.[Gr]) on $\mathbb{R}^{\mathrm{n}}$, i.e. $\mathbb{R}^{\mathrm{n}}$ together with $<,>$ and $\underline{\Delta}$ is a fixed oriented Euclidean space.

Each $J \in E\left(M, \mathbb{R}^{n}\right)$ and each $j \in E\left(\partial M, \mathbb{R}^{n}\right)$ yield Riemannian metrics $m(J)$ and $m(j)$ on $\mathrm{M}$ and $\partial \mathrm{M}$ respectively. These metrics are defined by 


$$
\mathrm{m}(J)(X, Y)=<\mathrm{d} J \mathrm{X}, \mathrm{d} J \mathrm{Y}\rangle, \quad \forall \mathrm{X}, \mathrm{Y} \in \Gamma \mathrm{TM}
$$

and

$$
m(j)(X, Y)=\langle\operatorname{djX}, \operatorname{dj} Y>, \quad \forall X, Y \in \Gamma T \partial M
$$

Here $\Gamma T Q$ denotes the collection of all smooth vector fields of any smooth manifold $Q$ (with or without boundary). Both $m(J)$ and $m(j)$ depend smoothly on its variables $\mathrm{J}$ and $\mathrm{j}$.

Associated with the metrics $m(J)$ and $m(j)$, we have the respective Levi-Civita connections $\nabla(J)$ on $M$ and $\nabla(j)$ on $\partial \mathrm{M}$. They are determined by

$$
\text { dJ } \nabla(J)_{X} Y=d(d J Y)(X), \quad \forall X, Y \in \Gamma T M
$$

and

$$
\operatorname{dj} \nabla(j)_{X} Y=d(d j Y)(X)-m(j)(W(j) X, Y) \cdot N(j), \quad \forall X, Y \in \Gamma T \partial M
$$

- By $W(j)$ we mean the Weingarten map given by

$$
\operatorname{dN}(\mathrm{j}) \mathrm{Z}=\operatorname{djW}(\mathrm{j}) \mathrm{Z}, \quad \forall \mathrm{Z} \in \Gamma \mathrm{TM}
$$

; where $N(j)$ is the unite normal vector field along $j$, for which ${ }^{*}{ }^{i} N(j) \underline{\Delta}$ determines the orientation class of $\partial \mathrm{M}$.

For any $J \in E\left(M, \mathbb{R}^{\mathrm{n}}\right)$ and any $\mathrm{j} \in \mathrm{E}\left(\mathrm{M}, \mathbb{R}^{\mathrm{n}}\right)$ let us denote by $\mu(J)$ and $\mu(\mathrm{j})$ the Riemannian volume form determined by $\mathrm{m}(\mathrm{J})$ and the orientation of $M$ respectively by $m(j)$ and the orientation of $\partial \mathrm{M}$. Let us denote by $\mathrm{N}$ the positively oriented unite normal vector field on $\partial \mathrm{M}$. This vector field depends on $m(J)$.

\section{Special one forms on $E\left(M, \mathbb{R}^{n}\right)$ and $E\left(M, \mathbb{R}^{n}\right) / \mathbb{R}^{n}$}

We will characterize the type of the material which constitutes the body $M$ in so far as it affects the work caused by an infinitesimal distortion of $M$ (cf.[He], [E,S], - $[\mathrm{Bi}],[\mathrm{Bi}, \mathrm{Sc}, \mathrm{So}])$. This idea is formalized by giving a smooth one-form on $\mathrm{E}\left(\mathrm{M}, \mathbb{R}^{\mathrm{n}}\right)$, i.e. a smooth map

$$
F: E\left(M, \mathbb{R}^{n}\right) \times C^{\infty}\left(M, \mathbb{R}^{n}\right) \rightarrow \mathbb{R}
$$


which varies linearly in the second argument. We interpret $F(J)(L)$ as the work done if $M$ is distorded by $L \in C^{\infty}\left(M, \mathbb{R}^{n}\right)$ at the configuration $J \in E\left(M, \mathbb{R}^{n}\right)$. We call the medium described by $\mathrm{F}$ a smoothly deformable medium.

Next we expose $\mathrm{F}$ to the translational symmetry and require that

$$
\mathrm{F}(\mathrm{J}+\mathrm{z})=\mathrm{F}(\mathrm{J}), \quad \forall \mathrm{J} \in \mathrm{E}\left(\mathrm{M}, \mathbb{R}^{\mathrm{n}}\right), \forall \mathrm{z} \in \mathbb{R}^{\mathrm{n}}
$$

This means that the work caused by physical processes under consideration does not depend on the particular location of $J(M)$ within $\mathbb{R}^{n}$. Moreover a constant distortion by any $\mathrm{z} \in \mathbb{R}^{\mathrm{n}}$ is supposed to cause no work. More formally this means that we impose the following restrictions on $F$ basic to our further development :

$$
\text { (ii.) } \mathrm{F}(J)(\mathrm{z})=0, \quad \forall J \in E\left(M, \mathbb{R}^{\mathrm{n}}\right), \forall \mathrm{z} \in \mathbb{R}^{\mathrm{n}} \text {. }
$$

- One-forms $\mathrm{F}$ on $\mathrm{E}\left(\mathrm{M}, \mathbb{R}^{\mathrm{n}}\right)$ satisfying (2.2) and (2.3) will be the basic ingredients of : our notion of constitutive laws. To implement the possibility of extracting force densities from our basic notion of work we need a little more structure associated with our forms satisfying (2.2) and (2.3). We will do this in the next paragraph.

But first let us state the following obvious lemma:

\section{Lemma 2.1 :}

A smooth one-form $F: E\left(M, \mathbb{R}^{n}\right) \times C^{\infty}\left(M, \mathbb{R}^{n}\right) \rightarrow \mathbb{R}$ satisfying (2.2) and (2.3)iff it is of the form $\mathrm{F}=\mathrm{d}^{*} \mathrm{~F}_{\mathbb{R}^{n}}$ that is

$$
\mathrm{F}(\mathrm{J})(\mathrm{L})=\mathrm{F}_{\mathbb{R}^{n}}(\mathrm{dJ})(\mathrm{dL}), \quad \forall \mathrm{J} \in \mathrm{E}\left(\mathrm{M}, \mathbb{R}^{\mathrm{n}}\right) \text { and } \forall \mathrm{L} \in \mathrm{C}^{\infty}\left(\mathrm{M}, \mathbb{R}^{\mathrm{n}}\right),
$$

where

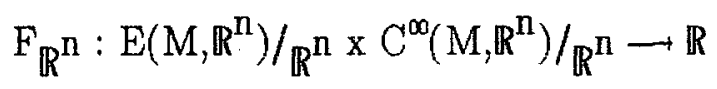

is a smooth one-form. 
In the last section we will study the effect of the rotational symmetry on our

special types of one-forms, the constitutive laws.

\section{The notion of a constitutive law, the Dirichlet integral}

The purpose of this section is to define what is meant by an integral representation of a one-form $F_{\mathbb{R}^{n}}$ on $E\left(M, \mathbb{R}^{n}\right)$ and in turn to define the notion of a constitutive law.

To obtain a tool general enough, we first will introduce a quadric structure on $E\left(M, \mathbb{R}^{n}\right) \times A^{1}\left(M, \mathbb{R}^{n}\right)$. We denote the collection of all smooth $\mathbb{R}^{m}$-valued one-forms of any smooth manifold $Q$ by $A^{1}\left(Q, \mathbb{R}^{m}\right)$ and equip with the $C^{\infty}$-topology in case $Q$ is compact.

Let $\gamma \in \mathrm{A}^{\mathrm{l}}\left(\mathrm{M}, \mathbb{R}^{\mathrm{n}}\right)$ and $\mathrm{J} \in \mathrm{E}\left(\mathrm{M}, \mathbb{R}^{\mathrm{n}}\right)$ be given. The two tensor $<\gamma, \mathrm{dJ}>$ determined by $\gamma$ and $J$ shall be given by $\langle\gamma \mathrm{X}, \mathrm{dJY}\rangle$ for all $\mathrm{X}, \mathrm{Y} \in \Gamma \mathrm{TM}$. This two tensor $:<\gamma, \mathrm{dJ}>$ yields a unique strong bundle map $\mathrm{A}(\gamma, \mathrm{dJ})$ of TM defined by

$$
<\gamma \mathrm{X}, \mathrm{d} J \mathrm{Y}\rangle=\mathrm{m}(\mathrm{J})(\mathrm{A}(\gamma, \mathrm{dJ}) \mathrm{X}, \mathrm{Y}), \quad \forall \mathrm{X}, \mathrm{Y} \in \Gamma \mathrm{TM}
$$

From this equation we read off :

$$
\gamma \mathrm{X}=\mathrm{dJA}(\gamma, \mathrm{dJ}) \mathrm{X}, \quad \forall \mathrm{X} \in \Gamma \mathrm{TM}
$$

For any pair of one-forms $\gamma_{1}, \gamma_{2} \in A^{\prime}\left(M, \mathbb{R}^{n}\right)$ and any embedding $J \in E\left(M, \mathbb{R}^{n}\right)$ we define the dot product of $\gamma_{1}$ and $\gamma_{2}$ relative to $J$ by

$$
\gamma_{1} \cdot \gamma_{2}:=\operatorname{tr} \mathrm{A}\left(\gamma_{1}, \mathrm{~d} J\right) \cdot \tilde{\mathrm{A}}\left(\gamma_{2}, \mathrm{dJ}\right)
$$

which is a smooth map on M. Here $\widetilde{A}\left(\gamma_{2}, \mathrm{dJ}\right)$ is the adjoint of $\mathrm{A}\left(\gamma_{2}, \mathrm{dJ}\right)$ formed fibre-wise with respect to $\mathrm{m}(\mathrm{J})$.

Associated with this product is a quadratic structure $G_{\mathbb{R}^{n}}(d J)$ on $A^{1}\left(M, \mathbb{R}^{n}\right)$ defined - by

$$
\mathrm{G}_{\mathbb{R}^{\mathrm{n}}(\mathrm{dJ})\left(\gamma_{1}, \gamma_{2}\right):=} \int_{\mathrm{M}} \gamma_{1} \cdot \gamma_{2} \mu(\mathrm{J})
$$


- As mentioned before $\mu(\mathrm{J})$ denotes the Riemannian volume form determined by

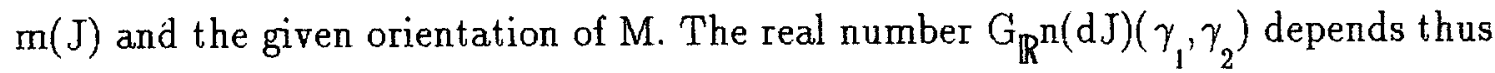
smoothly on all its variables $\mathrm{dJ}, \gamma_{1}$ and $\gamma_{2}$.

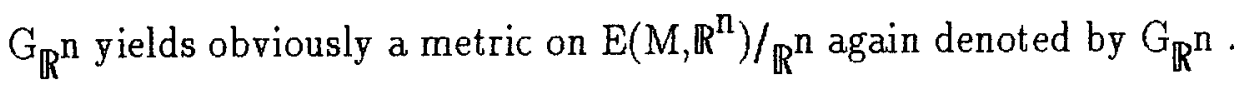

We say that $F_{\mathbb{R}^{n}}$, a one-form on $E\left(M, \mathbb{R}^{n}\right) / \mathbb{R}^{n}$, admits an integral repesentation if there exists a smooth map

$$
\alpha: \mathrm{E}\left(\mathrm{M}, \mathbb{R}^{\mathrm{n}}\right) \rightarrow \mathrm{A}^{1}\left(\mathrm{M}, \mathbb{R}^{\mathrm{n}}\right)
$$

called the kernel of $F_{\mathbb{R}^{n}}$, such that for any choices of $d J \in E\left(M, \mathbb{R}^{n}\right) / \mathbb{R}^{n}$ and $\mathrm{dL} \in \mathrm{C}^{\infty}\left(\mathrm{M}, \mathbb{R}^{\mathrm{n}}\right) / \mathbb{R}^{\mathrm{n}}$

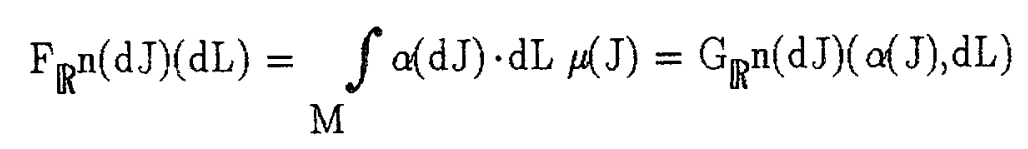

holds true.

We speak of a constitutive law $F$, if $F=d^{*} F_{\mathbb{R}^{n}}$ and $F_{\mathbb{R}^{n}}$ admits an integral representation with kernel $\alpha$.

To discuss the uniqueness of the kernel $\alpha$, associated with a constitutive law, we first prove the following :

\section{Theorem 3.1:}

Let $\gamma \in \mathrm{A}^{1}\left(M, \mathbb{R}^{\mathrm{n}}\right)$ and $J \in E\left(M, \mathbb{R}^{\mathrm{n}}\right)$. There exists a uniquely determined differential $\mathrm{d} \mathfrak{H} \in \mathrm{C}^{\infty}\left(\mathrm{M}, \mathbb{R}^{\mathrm{n}}\right) / \mathbb{R}^{\mathrm{n}}$ called the exact part of $\gamma$ and a uniquely determined $\beta \in \mathrm{A}^{1}\left(\mathbb{M}, \mathbb{R}^{\mathrm{n}}\right)$ such that

$$
\gamma=\mathrm{d} \mathfrak{H}+\beta
$$

where the exact part of $\beta$ vanishes. Both $\mathrm{d} \mathfrak{H}$ and $\beta$ depend smoothly on J. If $\mathfrak{H}\left(\mathrm{p}_{0}\right)$ for some $\mathrm{p}_{0} \in \mathrm{M}$ is kept constant in $\mathrm{J}$, then also $\mathfrak{H}$ varies smoothly in $J$. 
- Proof :

- We will exhibit the proof to some details, in order to reveal the surrounding structure, which will allow us to handle the integral representation introduced in (3.5). First let us construct $\mathfrak{H}$ and $\beta$. To this end we fix a basis $e_{1}, \ldots, e_{n}$ on $\mathbb{R}^{n}$, orthonormal with respect to $<,>$. With these data we may write

$$
x(\mathrm{X})=\sum_{\mathrm{r}=1}^{\mathrm{n}} \gamma^{\mathrm{r}}(\mathrm{X}) \mathrm{e}_{\mathrm{r}}, \quad \forall \mathrm{X} \in \Gamma \mathrm{TM}
$$

with $\gamma^{\Gamma} \in A^{1}\left(M, \mathbb{R}^{n}\right)$ for all $r=1, \ldots, n$. Since for each $r$

$$
\gamma^{\mathrm{I}}(\mathrm{X})=\mathrm{m}(\mathrm{J})\left(\mathrm{Y}^{\mathrm{I}}, \mathrm{X}\right), \quad \forall \mathrm{X} \in \Gamma \mathrm{TM}
$$

holds true for a well defined $\mathrm{Y}^{\mathrm{I}} \in \Gamma \mathrm{TM}$, we find due to Hodge's decomposition (cf.[A,M,R]) a function $\tau^{\mathrm{I}} \in \mathrm{C}^{\infty}\left(\mathrm{M}, \mathbb{R}^{\mathrm{n}}\right)$ and a uniquely determined vector field $Y_{0}^{I} \in \Gamma T M$ such that the following three equations are satisfied

$$
\mathrm{Y}^{\mathrm{r}}=\operatorname{grad}_{J^{\mathrm{T}}} \tau^{\mathrm{T}} \mathrm{Y}_{0}^{\mathrm{r}}
$$

and

$$
\begin{gathered}
\operatorname{div}_{J} Y_{0}^{r}=0 \\
\text { together with the boundary condition } \\
m(J)\left(Y_{0}^{r}, N\right)=0 \text { along } \partial \mathrm{M}
\end{gathered}
$$

Here the indices $J$ in $\operatorname{grad}_{J}$ and $\operatorname{div}_{J}$ mean that the respective operations are formed with respect to $\mathrm{m}(\mathrm{J})$. This decomposition is obtained by solving the following von Neumann problem

$$
\Delta(J) \tau^{\mathrm{T}}=-\operatorname{div} Y^{\mathrm{I}}
$$

- with the boundary condition

$$
\mathrm{d} \tau^{\mathrm{I}}(\mathrm{N})=\mathrm{m}(\mathrm{J})\left(\mathrm{Y}^{\mathrm{I}}, \mathrm{N}\right)
$$

This problem has, according to [Hö], a solution $\tau^{\Gamma}$ unique up to a constant. 
* The desired function $\mathfrak{H}$ and the form $\beta$ are defined by

$$
\mathfrak{H}:=\sum_{\mathrm{r}} \tau^{\mathrm{r}} \cdot \mathrm{e}_{\mathrm{r}}
$$

and

$$
\not(X):=\underset{\Gamma}{\Sigma} m(J)\left(Y_{0}{ }^{\mathrm{I}}, \mathrm{X}\right), \quad \forall X \in \Gamma \mathrm{TM}
$$

respectively. It is a matter of routine to show that $\mathrm{d} \mathfrak{H}$ and $\beta$ do not depend on the basis chosen. A somewhat involved matter is to show the smoothness properties. With these notions we immediately deduce (3.6). One easily verifies that the exact part of $\beta$ vanishes.

Some of the calculation made in the proof above allow us to look at $G_{\mathbb{R}} n(d J)$ from another angle. Given $\gamma \in \mathrm{A}^{1}\left(\mathrm{M}, \mathbb{R}^{\mathrm{n}}\right)$ and $J \in \mathrm{E}\left(\mathrm{M}, \mathbb{R}^{\mathrm{n}}\right)$ we have according to (3.7) and (3.8) in above proof

$$
\eta(X)=\mathrm{d} J A(\gamma, \mathrm{d} J) X=\sum_{\mathrm{r}=1}^{\mathrm{n}} \mathrm{m}(J)\left(\mathrm{Y}^{\mathrm{r}}, \mathrm{X}\right) \mathrm{e}_{\mathrm{r}}, \quad \forall \mathrm{X} \in \Gamma \mathrm{TM}
$$

Let us denote $(d J)^{-1} e_{r}$ by $E_{r}$, for all $r=1, \ldots, n$. Then we read off from the equation (3.15) that

$$
\mathrm{Y}^{\mathrm{r}}=\tilde{\mathrm{A}}(\gamma, \mathrm{dJ}) \mathrm{E}_{\mathrm{r}}, \quad \forall \mathrm{r}=1, \ldots, \mathrm{n}
$$

holds true. This remark yields the following observation :

\section{Proposition 3.2 :}

Given $\gamma_{1}, \gamma_{2} \in \mathrm{A}^{\prime}\left(\mathrm{M}, \mathbb{R}^{\mathrm{n}}\right), \mathrm{J} \in \mathrm{E}\left(\mathrm{M}, \mathbb{R}^{\mathrm{n}}\right)$ and a fixed basis $\mathrm{e}_{1}, \ldots, \mathrm{e}_{\mathrm{n}}$ on $\mathbb{R}^{\mathrm{n}}$ orthonormal with respect to $<,>$, then there exist two sets

$$
\mathrm{Y}_{1}^{1}, \ldots, \mathrm{Y}_{1}^{\mathrm{n}} \text { and } \mathrm{Y}_{2}^{1}, \ldots, \mathrm{Y}_{2}^{\mathrm{n}}
$$


of vector fields in ГTM, such that

$$
\gamma_{1} \cdot \gamma_{2}=\sum_{\mathrm{r}=1}^{\mathrm{n}} \mathrm{m}(\mathrm{J})\left(\mathrm{Y}_{1}^{\mathrm{I}}, \mathrm{Y}_{2}^{\mathrm{I}}\right)
$$

and hence

$$
\mathrm{G}_{\mathbb{R}^{\mathrm{n}}(\mathrm{dJ})\left(\gamma_{1}, \gamma_{2}\right)}:=\int_{\mathrm{M}} \gamma_{1} \cdot \gamma_{2} \mu(\mathrm{J})=\sum_{\mathrm{r}=1}^{\mathrm{n}} \int_{\mathrm{M}} \mathrm{m}(\mathrm{J})\left(\mathrm{Y}_{1}^{\mathrm{I}}, \mathrm{Y}_{2}{ }_{2}^{\mathrm{I}}\right) \mu(\mathrm{J})
$$

If in addition $\gamma_{1}=\mathrm{d} \mathfrak{H}$ for some $\mathfrak{H} \in C^{\infty}\left(M, \mathbb{R}^{\mathrm{n}}\right)$ then $G_{\mathbb{R}^{n}}(\mathrm{~d} J)\left(\mathrm{d} \mathfrak{H}, \gamma_{2}\right)=0$ provided that the exact part of $\gamma_{2}$ vanishes.

\section{Proof :}

Let $Y_{i}{ }^{r} \in \Gamma T M$ for $r=1, \ldots, n$ and $i=1,2$ be as in (3.8). Then

$$
\begin{aligned}
\gamma_{1} \cdot \gamma_{2}=\operatorname{tr} \mathrm{A}\left(\gamma_{1}, \mathrm{dJ}\right) \cdot \tilde{\mathrm{A}}\left(\gamma_{2}, \mathrm{dJ}\right)=\sum_{\mathrm{r}=1}^{\mathrm{n}} \mathrm{m}(\mathrm{J})\left(\mathrm{A}\left(\gamma_{1} \mathrm{dJ}\right) \cdot \tilde{\mathrm{A}}\left(\gamma_{2}, \mathrm{dJ}\right) \mathrm{E}_{\mathrm{r}}, \mathrm{E}_{\mathrm{r}}\right) \\
=\sum_{\mathrm{r}=1}^{\mathrm{n}} \mathrm{m}(\mathrm{J})\left(\mathrm{Y}_{1}{ }_{1}^{\mathrm{r}}, \mathrm{Y}_{2}{ }_{2}\right)
\end{aligned}
$$

establishing (3.17). To show the last part of the proposition we use Gauss' theorem under the three assumptions that $\mathfrak{H}=\sum_{\mathrm{I}=1}^{\mathrm{n}} \tau^{\mathrm{r}} \mathrm{e}_{\mathrm{r}}$ and $\operatorname{div}_{\mathrm{J}} \mathrm{Y}_{0}^{\mathrm{r}}=0$ as well as $\mathrm{m}(\mathrm{J})\left(\mathrm{Y}_{0}^{\mathrm{T}}, \mathrm{N}\right)=0$ :

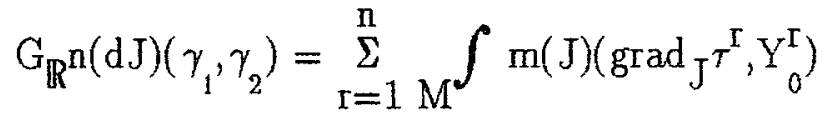

$$
\begin{aligned}
& =\sum_{\mathrm{I}=1 \mathrm{M}^{\mathrm{N}}}^{\mathrm{n}} \int \mathrm{d} \tau^{\mathrm{T}}\left(\mathrm{Y}_{0}^{\mathrm{T}}\right) \mu(\mathrm{J})
\end{aligned}
$$

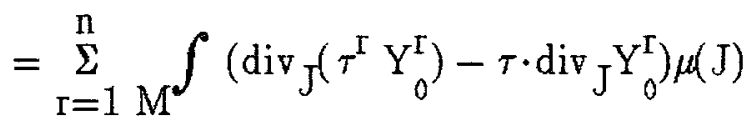

$$
\begin{aligned}
& =\sum_{\mathrm{r}=1}^{\mathrm{n}} \int_{\mathrm{M}} \mathrm{m}(\mathrm{J})\left(\tau^{\mathrm{T}} \mathrm{Y}_{0}^{\mathrm{T}}, \mathrm{N}\right) \mu(\mathrm{J}) \\
& =0 \text {. }
\end{aligned}
$$


In case $\gamma_{1}$ and $\gamma_{2}$ in the above proposition are exact, then the respective vector fields in (3.20) are gradients. Hence the right hand side of the integral in (3.18) is the classical Dirichlet integral (cf.[J]) for $\mathbb{R}^{n}$-valued functions. We call the middle integral in (3.18), therefore the Dirichlet integral of any two smooth $\mathbb{R}^{\mathrm{n}}$-valued forms $\gamma_{1}, \gamma_{2}$ relative to $\mathrm{J} \in \mathrm{E}\left(\mathrm{M}, \mathbb{R}^{\mathrm{n}}\right)$.

Proposition 3.2 also shows that the integral kernel of a constitutive law is not unique at all. We may add to any kernel a map which assumes as its values, one-forms of which the exact part vanishes. However the following theorem guaranties us a uniqueness of a very specific type of kernel. The proof of the following theorem is a matter of routine :

\section{Theorem 3.3 :}

Let $\mathrm{F}$ be a constitutive law with integral kernel. There exists a unique smooth map

$$
\alpha: E\left(M, \mathbb{R}^{n}\right) /_{\mathbb{R}^{n}} \rightarrow C^{\infty}\left(M, \mathbb{R}^{n}\right) \mathbb{R}_{\mathbb{R}^{n} \subset A^{1}\left(M, \mathbb{R}^{n}\right)}
$$

such that for any $J \in E\left(M, \mathbb{R}^{n}\right)$ and any $L \in C^{\infty}\left(M, \mathbb{R}^{n}\right)$

$$
F(J)(L)=\int_{M} \alpha(d J) \cdot d L \mu(J)=G_{\mathbb{R}^{n}}(\mathrm{dJ})(\alpha(\mathrm{dJ}), \mathrm{dL})
$$

holds true. In fact there is a unique smooth map

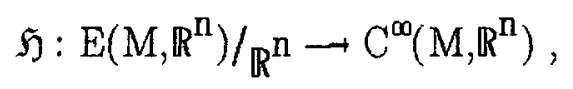

satisfying the following two equations

$$
\alpha(\mathrm{dJ})=\mathrm{d} \mathfrak{H}(\mathrm{dJ}), \quad \forall \mathrm{dJ} \in \mathrm{E}\left(\mathrm{M}, \mathbb{R}^{\mathrm{n}}\right) /_{\mathbb{R}^{\mathrm{n}}}
$$

and

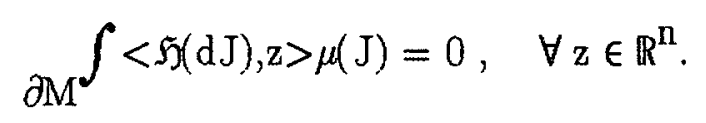


Equation (3.22) together with (3.23) show that $\mathrm{d} \mathfrak{H}$ is the generalized gradient of $F_{\mathbb{R}^{n}}$ with respect to $G_{\mathbb{R}}{ }^{n}$.

It is this type of gradient mentioned above which characterizes the material.

Clearly each constitutive law F forms via $\alpha$ and equation (3.1) a tensor on $\mathrm{M}$ given by

$$
\begin{aligned}
\mathrm{T}(J)(X, Y):= & <\alpha(\mathrm{dJ}) \mathrm{X}, \mathrm{d} J Y> \\
& =\mathrm{m}(J)(\mathrm{dJA}(\alpha(\mathrm{dJ}), \mathrm{dJX}), \mathrm{Y}\rangle, \quad \forall \mathrm{X}, \mathrm{Y} \in \Gamma T \mathrm{TM}
\end{aligned}
$$

Vice versa each two-tensor depending smoothly on dJ defines a kernel of a constitutive law. We call $T(J)$ the stress tensor at the configuration $J$.

$T(J)$ splits into a symmetric and a skew symmetric part. We will investigate at the end of these notes under what conditions on $\mathfrak{d} \mathfrak{H}$ the skew symmetric part vanishes.

\section{Force densities associated with constitutive laws}

The purpose of this section is to associate with any constitutive law at any configuration some well defined force densities, one acting upon the whole body, and an extra one acting upon the boundary only. Vice versa any given pair of such force densities satisfying an integrability condition will be obtained via a suitable constitutive law.

Throughout this section $\mathrm{F}$ is a constitutive law, its kernel is called $\alpha$. By the previous theorem we may assume that $\alpha\left(E\left(M, \mathbb{R}^{n}\right) / \mathbb{R}^{n}\right) \subset C^{\infty}\left(M, \mathbb{R}^{n}\right) / \mathbb{R}^{n}$.

To construct the force densities mentioned we use $\mathrm{F}$ in the form

$$
\mathrm{F}(\mathrm{J})(\mathrm{L})=\int_{\mathrm{M}} \operatorname{tr} \mathrm{A}(\alpha(\mathrm{dJ}), \mathrm{dJ}) \cdot \tilde{\mathrm{A}}(\mathrm{dL}, \mathrm{dJ}) \mu(J)
$$

holding for any of the variables of $F$. Representing any $L \in C^{\infty}\left(M, \mathbb{R}^{n}\right)$ relative to a given $J \in E\left(M, \mathbb{R}^{n}\right)$ in the form 


$$
L=d J X(L, J)
$$

with a unique $X(L, J) \in \Gamma T M$ we derive

$$
\mathrm{dL} X=\mathrm{d} J \nabla(J)_{X} \mathrm{X}(\mathrm{L}, J), \quad \forall \mathrm{X} \in \Gamma \mathrm{TM}
$$

and hence obtain immediately

$$
\mathrm{A}(\mathrm{dL}, \mathrm{d} J)=\nabla(J) X(L, J), \quad \forall \mathrm{L} \in \mathrm{C}^{\infty}\left(\mathrm{M}, \mathbb{R}^{\mathrm{n}}\right)
$$

Thus if $e_{1}, \ldots, e_{n}$ is a orthonormal basis of $\mathbb{R}^{n}$ for which we let $E_{r} \in \Gamma T M$ again be given by $d J E_{r}=e_{r}$ for $r=1, \ldots, n$ then

$$
F(J)(L)=\sum_{r=1}^{n} \int m(J)\left(\left.\tilde{A}(\alpha(d J), d J) \cdot \nabla(J)\right|_{E_{r}} X(L, J), E_{r}\right) \mu(J)
$$

Let us introduce the notion $\operatorname{div}_{\mathrm{J}} \mathrm{T}$, the divergence of a strong bundle endomorphism $\mathrm{T}$ of TM by

$$
\operatorname{div}_{J} T:=\sum_{r=1}^{n} \nabla(J)_{E_{\mathrm{r}}}(T)\left(\mathrm{E}_{\mathrm{r}}\right)
$$

This notion does not depend of the basis chosen.Equation (4.6) together with (4.5) imply

$$
\begin{aligned}
F(J)(L)= & \int_{M} \operatorname{div}_{J}(\tilde{A}(\alpha(d J), d J) X(L, J)) \mu(J) \\
& -\int_{M} m(J)\left(\operatorname{div}_{J} A(\alpha(d J), d J), X(L, J)\right) \mu(J) .
\end{aligned}
$$

To bring these formulas in a more familiar form we introduce the notions of $\Delta(J) \mathrm{K}$ and $\Delta(J) \gamma$, the Laplacian for any $\mathrm{K} \in \mathrm{C}^{\infty}\left(\mathrm{M}, \mathbb{R}^{\mathrm{n}}\right)$ and any $\gamma \in \mathrm{A}^{\mathrm{I}}\left(\mathrm{M}, \mathbb{R}^{\mathrm{n}}\right)$. In doing so we follow [Mat]. We begin with the definition of $\delta$ by letting

$$
\delta \mathrm{K}=0 .
$$

If $\gamma \in \mathrm{A}^{\mathrm{l}}\left(\mathrm{M}, \mathbb{R}^{\mathrm{m}}\right)$ for some natural number $\mathrm{m}$, we set 


$$
\delta \gamma=\sum_{\mathrm{r}=1}^{\mathrm{n}} \nabla(\mathrm{J})_{\mathrm{E}_{\mathrm{I}}}(\gamma)\left(\mathrm{E}_{\mathrm{I}}\right)
$$

Clearly

$$
\delta \gamma=-\operatorname{div}_{J} Y
$$

if

$$
\chi(X)=m(J)(Y, X), \quad \forall X \in \Gamma T M
$$

$\Delta(\mathrm{J})$ is then defined by

$$
\Delta(\mathrm{J}):=\mathrm{d} \delta+\delta \mathrm{d}
$$

Consequently we have

$$
\Delta(J) K=\delta \mathrm{dK}=-\sum_{\mathrm{r}=1}^{\mathrm{n}} \nabla(J)_{\mathrm{E}_{\mathrm{r}}}(\mathrm{dK})\left(\mathrm{E}_{\mathrm{r}}\right)
$$

and therefore we verify the following relation :

$$
\begin{aligned}
\Delta(J) K & =-\left(\sum_{\Gamma=1}^{n} \mathrm{~d}\left(\mathrm{dJ} A\left(\mathrm{dK}, \mathrm{dJ} \mathrm{E}_{\mathrm{r}}\right)\right)\left(\mathrm{E}_{\mathrm{r}}\right)-\mathrm{dJ} A(\mathrm{dK}, \mathrm{dJ})\left(\nabla(J)_{\mathrm{E}_{\mathrm{I}}} \mathrm{E}_{\mathrm{I}}\right)\right) \\
& =-\sum_{\mathrm{I}=1}^{\mathrm{n}} \mathrm{dJ} \nabla(J)_{\mathrm{E}_{\mathrm{I}}}(\mathrm{A}(\mathrm{dK}, \mathrm{dJ})) \mathrm{E}_{\mathrm{I}} \\
& =-\mathrm{dJ} \operatorname{div} \mathrm{J}(\mathrm{dK}, \mathrm{d} J)
\end{aligned}
$$

Hence equation (4.7) turns into

$$
\begin{array}{r}
F(J)(L)=\int \operatorname{Miv}_{J}(\tilde{A}(\alpha(d J), d J) X(L, J)) \mu(J) \\
+\int_{M} \int<\Delta(J) \mathfrak{S}(\mathrm{d} J), L>\mu(J),
\end{array}
$$

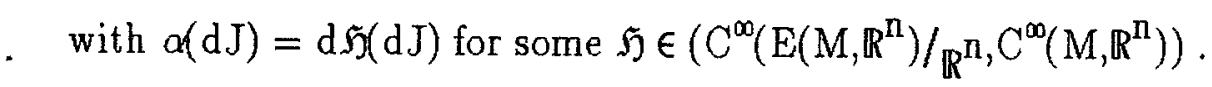

- Using Gauss'theorem we derive with the help of theorem 3.3 the following 


\section{Proposition 4.1 :}

Let $F$ be a constitutive law. Then for each $J \in E\left(M, \mathbb{R}^{n}\right)$ there exists a smooth map

$$
\mathfrak{H}: \mathrm{E}\left(\mathrm{M}, \mathbb{R}^{\mathrm{n}}\right) \rightarrow \mathrm{C}^{\infty}\left(\mathrm{M}, \mathbb{R}^{\mathrm{n}}\right)
$$

uniquely determined up to a smooth map from $E\left(M, \mathbb{R}^{n}\right)$ into $\mathbb{R}^{n}$ for which

$$
\begin{aligned}
\mathrm{F}(J)(\mathrm{L}) & =\int<\Delta(J) \mathfrak{H}(\mathrm{dJ}), \mathrm{L}>\mu(\mathrm{J}) \\
+ & \int<\mathrm{d} \mathfrak{H}(\mathrm{dJ})(\mathrm{N}), \mathrm{L}>\mathrm{i}_{\mathrm{N}} \mu(\mathrm{J})
\end{aligned}
$$

and in turn a Green's equation

$$
\begin{aligned}
& \mathrm{M}^{\int<\Delta(\mathrm{J}) \mathfrak{H}(\mathrm{dJ}), \mathrm{L}>\mu(\mathrm{J})-{ }_{\mathrm{M}} \int<\mathfrak{H}(\mathrm{dJ}), \Delta(\mathrm{J}) \mathrm{L}>\mu(\mathrm{J})} \\
& ={ }_{\partial \mathrm{M}} \int\left\langle\mathrm{dL}(\mathrm{N}), \mathfrak{H}(\mathrm{dJ})>\mathrm{i}_{\mathrm{N}} \mu(\mathrm{J})-{ }_{\partial \mathrm{M}} \int<\mathrm{d} \mathfrak{H}(\mathrm{dJ})(\mathrm{N}), \mathrm{L}>\mathrm{i}_{\mathrm{N}} \mu(\mathrm{J})\right.
\end{aligned}
$$

: are valued for all variables of $\mathrm{F}$. Here $\mathrm{i}_{\mathrm{N}} \mu(\mathrm{J})$ is the volume element on $\partial \mathrm{M}$ defined : by $\mu(J)$ and $N$, the positively oriented unite normal vector field along $\partial \mathrm{M} \subset \mathrm{M}$.

The developments made so far show that any characteristic of the material formulated via our notion of a constitutive law are encoded in the map $\mathfrak{H}$. We call this map $\mathfrak{H}$ therefore a constitutive map.

The above proposition motivates us to set for any $J \in E\left(M, \mathbb{R}^{n}\right)$

and

$$
\Phi(J):=\Delta(J) \mathfrak{H}(\mathrm{dJ})
$$

$$
\varphi(\mathrm{d} J):=\mathrm{d} \mathfrak{H}(\mathrm{d} J)(\mathrm{N})
$$

with $\mathfrak{H}(\mathrm{dJ})$ as in (4.15).

. We call the maps $\Phi$ and $\varphi$ the force densities associated with $F$.

- To prove the following theorem is now a matter of routine again. 


\section{Theorem 4.2:}

Every constitutive law $F \in A^{1}\left(E\left(M, \mathbb{R}^{n}\right), \mathbb{R}\right)$ admits a smooth constitutive map

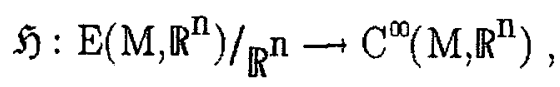

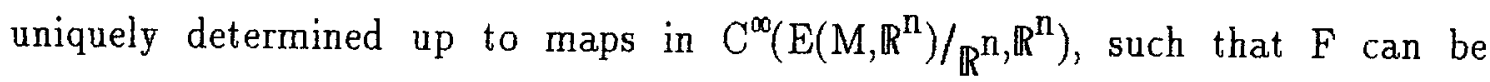
expressed by

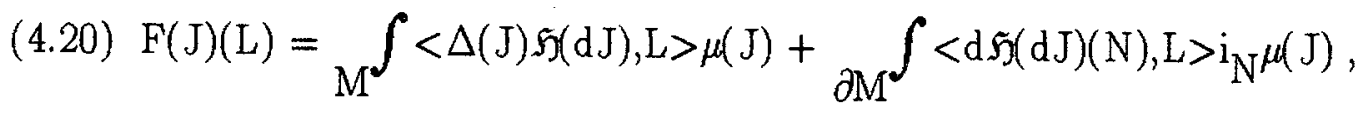

for each $J \in E\left(M, \mathbb{R}^{n}\right)$ and each $L \in C^{\infty}\left(M, \mathbb{R}^{n}\right)$. For all $J \in E\left(M, \mathbb{R}^{n}\right)$ the map $\mathfrak{H}$ defines the force densities

$$
\Phi(\mathrm{d} J)=\Delta(J) \mathfrak{H}(\mathrm{dJ})
$$

: and

$$
\varphi(\mathrm{dJ})=\mathrm{d} \mathfrak{H}(\mathrm{d} J)(\mathrm{N})
$$

with the following property :

$$
0={ }_{\mathrm{M}} \int\left\langle\Phi(\mathrm{d} J), \mathrm{z}>\mu(J)+{ }_{\partial \mathrm{M}} \int\langle\varphi(\mathrm{d} J), \mathrm{z}) \mathrm{i}_{N} \mu(J), \forall \mathrm{z} \in \mathbb{R}^{\mathrm{n}}\right.
$$

Given vice versa two smooth maps

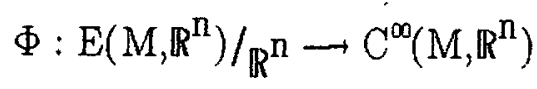

and

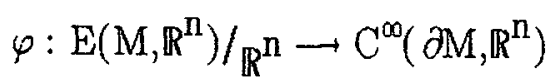

for which the integrability condition (4.23) holds. Then there exists a smooth map

$$
\mathfrak{H}: E\left(M, \mathbb{R}^{n}\right) / \mathbb{R}^{n} \rightarrow C^{\infty}\left(M, \mathbb{R}^{n}\right)
$$

such that $\mathfrak{H}(\mathrm{d} J)$ is uniquely determined up to a constant for each $J \in E\left(M, \mathbb{R}^{\mathrm{n}}\right)$ and - which satisfies (4.21) and (4.22). Moreover $\mathfrak{H}$ is a constitutive map for the constitutive law $\mathrm{F}$ given by 
(4.26) $\quad F(J)(L)=\int_{M} \int\left\langle\Phi(d J), L>\mu(J)+\int_{\partial M} \int\left\langle\varphi(d J), L>i_{N} \mu(J)\right.\right.$,

for all $J \in E\left(M, \mathbb{R}^{n}\right)$ and for all $L \in C^{\infty}\left(M, \mathbb{R}^{n}\right)$.

\section{Remark 4.3}

This theorem shows us that our notion of constitutive laws based on translational invariance are equivalent with the $\mathbb{R}^{n}$-valued solution of von Neumann problems formulated on $M$ and hence are equivalent with a pair of force densities satisfying the integrability condition (4.23). which is obviously the analogon of (2.3).

Moreover if for each $J \in E\left(M, \mathbb{R}^{n}\right)$ the one form $\alpha(d J)$ splits as

$$
\alpha(\mathrm{d} J)=\mathrm{d} \mathfrak{H}(\mathrm{dJ})+\beta(\mathrm{dJ})
$$

with $\mathrm{d} \mathfrak{H}(\mathrm{dJ})$ the exact part of $\alpha(\mathrm{dJ})$, then both $\alpha(\mathrm{dJ})$ and $\mathrm{d} \mathfrak{H}(\mathrm{dJ})$ determine the - same force densities!

\section{Constitutive laws for the boundary}

The task in this section is to study constitutive laws for the boundary, that is for a deformable medium forming a skin of which the underlying point set is the ( $\mathrm{n}-1$ )-dimensional manifold $\partial \mathrm{M}$. This skin, formed by a deformable material, will be studied on its own and is not regarded as boundary of some body. In doing so, we first formulate in analogy to sections two and three what is meant by a constitutive law for a skin. Also in this case the translational invariance and an appropriate integral representation of one-forms describing the work of the material subjected to distortions is the essential tool.

Let us recall that the open set $\mathrm{O}_{\partial} C \mathrm{E}\left(\partial \mathrm{M}, \mathbb{R}^{\mathrm{n}}\right)$ is the collection of all $\mathrm{J} \mid \partial \mathrm{M}$ with - $J \in E\left(M, \mathbb{R}^{\mathrm{n}}\right)$. The constitutive laws to introduce will be formulated on any open set $O \subset E\left(M, \mathbb{R}^{n}\right)$ and will later be specified on $\mathrm{O}_{\partial}$.

At the very first we introduce the notion corresponding to the Dirichlet integral : Given any $l \in C^{\infty}\left(\partial M, \mathbb{R}^{\mathrm{n}}\right)$ and any $j \in \mathrm{E}\left(\partial \mathrm{M}, \mathbb{R}^{\mathrm{n}}\right)$ then for all $\mathrm{X}, \mathrm{Y} \in \Gamma \mathrm{T} \partial \mathrm{M}$ 


$$
<\text { dl } X, d j Y>=m(j)(A(d l, d j) X, Y)
$$

holds for some smooth strong bundle-endomorphism $\mathrm{A}(\mathrm{dl}, \mathrm{dj})$ of $\mathrm{T} \partial \mathrm{M}$. Moreover there is a uniquely defined smooth map

$$
\mathrm{c}(\mathrm{dl}, \mathrm{dj}): \partial \mathrm{M} \rightarrow \mathrm{so}(\mathrm{n})
$$

with so( $\mathrm{n})$ the linear space of all skew maps of $\left(\mathbb{R}^{\mathrm{n}},<,>\right)$, satisfying the following two conditions

$$
c(d l, d j) d j\left(T_{p} \partial M\right) \subset \mathbb{R} \cdot N(j)(p), \quad \forall p \in \partial M
$$

and

$$
c(d l, d j) N(j)(p) c d j_{p} T_{p} \partial M, \quad \forall p \in \partial M
$$

and such that the equation

$$
d l X=c(d j, d l) d j X+d j A(d l, d j) X
$$

: holds true for any $\mathrm{X} \in \Gamma \mathrm{TTM}$. We refer to $[\mathrm{Bi}, \mathrm{Sn}, \mathrm{Fi}]$ or $[\mathrm{Bi}, \mathrm{Sc}, \mathrm{So}]$ for more details. Based on (5.4) we introduce $U(\mathrm{dl}, \mathrm{dj})$ by

$$
c(d l, d j) N(j)=d j U(d l, d j) .
$$

This vector field $U(d l, d j) \in \Gamma T \partial M$ is obviously uniquely determined.

Splitting $A(d l, d j)$ into its skew- respectively selfadjoint parts $C(d l, d j)$ and $B(d l, d j)$ formed pointwise with respect to $m(j)$ we end up with

$$
d l=c(d l, d j) \cdot d j+d j(C(d l, d j)+B(d l, d j)) .
$$

This decomposition relative to any $\operatorname{dj} \in \mathrm{E}\left(\partial \mathrm{M}, \mathbb{R}^{\mathrm{n}}\right)$ generalizes in the obvious way to any $\gamma \in \mathrm{A}^{\mathrm{l}}\left(\partial \mathrm{M}, \mathbb{R}^{\mathrm{n}}\right)$ and reads in this case as

$$
\gamma=c(\gamma, \mathrm{dj}) \cdot \mathrm{dj}+\mathrm{dj}(\mathrm{C}(\gamma, \mathrm{dj})+\mathrm{B}(\gamma, \mathrm{dj}))
$$

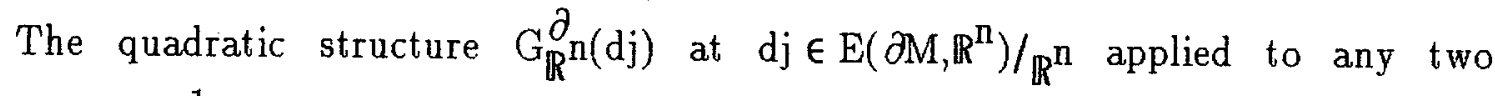
$\gamma_{1}, \gamma_{2} \in \mathrm{A}^{1}\left(\partial \mathrm{M}, \mathbb{R}^{\mathrm{n}}\right)$ is defined by integrating the function 
(5.8)

$$
\begin{aligned}
\gamma_{1} \cdot \gamma_{2}:=-\frac{1}{2} & \operatorname{trc}\left(\gamma_{1}, \mathrm{dj}\right) \cdot \mathrm{c}\left(\gamma_{2}, \mathrm{dj}\right) \\
& -\operatorname{tr} C\left(\gamma_{1}, \mathrm{dj}\right) \cdot C\left(\gamma_{2}, \mathrm{dj}\right) \\
& +\operatorname{tr} B\left(\gamma_{1}, \mathrm{dj}\right) \cdot B\left(\gamma_{2}, \mathrm{dj}\right)
\end{aligned}
$$

with respect to $\mu(\mathrm{j})$, that is, it is defined by

$$
\begin{aligned}
& \mathrm{G}_{\mathbb{R}^{\mathrm{n}}(\mathrm{dj})\left(\gamma_{1}, \gamma_{2}\right):={ }_{\mathrm{M}} \gamma_{1} \cdot \gamma_{2} \mu(\mathrm{j})}
\end{aligned}
$$

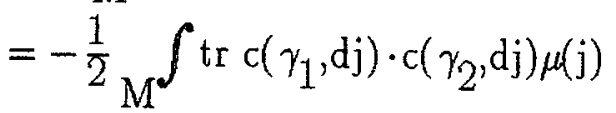

$$
\begin{aligned}
& { }_{\mathrm{M}^{\mathrm{S}}} \operatorname{tr} \mathrm{tr}\left(\gamma_{1}, \mathrm{dj}\right) \cdot \mathrm{C}\left(\gamma_{2}, \mathrm{dj}\right) \mu(\mathrm{j}) \\
& +\int_{M}^{\operatorname{tr} B\left(\gamma_{1}, d j\right) \cdot B\left(\gamma_{2}, d j\right) \mu(j) .}
\end{aligned}
$$

$G_{\mathbb{R}^{n}}^{\partial}$ obviously yields a metric on $E\left(M, \mathbb{R}^{n}\right)$. Let $O \subset E\left(\partial M, \mathbb{R}^{n}\right)$ be any open set.

- We now define a constitutive law $\mathrm{F}_{\partial}$ on $\mathrm{O}$ in analogy to section two, that is we : require

$$
\mathrm{F}_{\partial}=\mathrm{d}^{*} \mathrm{~F}_{\mathbb{R}^{n}}^{n^{\prime}}
$$

to hold for some one-form $\mathrm{F}_{\mathbb{R}^{\mathrm{n}}}^{\partial}$ on $O / \mathbb{R}^{\mathrm{n}}$ and demand furthermore that for some $\alpha \in C^{\infty}\left(O, A^{\prime}\left(\partial M, \mathbb{R}^{n}\right)\right)$ the following equation is valid

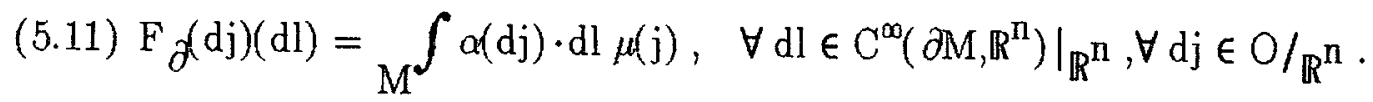

We introduce for any $\mathrm{j} \in \mathrm{E}\left(\partial \mathrm{M}, \mathbb{R}^{\mathbf{n}}\right)$ the Laplacian $\Delta(\mathrm{j})$ accordingly to (4.11) but require that $\mathrm{E}_{\mathrm{s}}$ in this case is a moving frame on $\partial \mathrm{M}$.

With this notion at hand the constitutive laws on $O$ are characterized in details in the next theorem :

\section{Theorem 5.1:}

Let $F_{\partial}$ be a constitutive law on any open set $O \subset E\left(\partial M, \mathbb{R}^{n}\right)$. The following are then - equivalent : 
(i.) $F_{\partial}$ admits a kernel $\alpha \in \mathrm{C}^{\infty}\left(O / \mathbb{R}^{n}, A^{1}\left(\partial M, \mathbb{R}^{n}\right)\right)$.

(ii.) There is a smooth map $\mathfrak{h} \in C^{\infty}\left(O / \mathbb{R}^{n}, C^{\infty}\left(\partial M, \mathbb{R}^{n}\right)\right)$ uniquely determined up to maps in $\mathrm{C}^{\infty}\left(O / \mathbb{R}^{n}, \mathbb{R}^{n}\right)$, such that

$$
\begin{array}{ll}
\mathrm{F}_{\partial j}(\mathrm{j})(\mathrm{l})={ }_{\partial \mathrm{M}}^{\int \mathrm{d} h(\mathrm{dj}) \cdot \mathrm{dl} \mu(\mathrm{j}),} \quad & \forall \mathrm{j} \in \mathrm{O}, \\
\text { and } \quad \forall \mathrm{l} \in \mathrm{C}^{\infty}\left(\partial \mathrm{M}, \mathbb{R}^{\mathrm{n}}\right) .
\end{array}
$$

(iii.) There is a smooth map $\mathfrak{h} \in \mathrm{C}^{\infty}\left(O / \mathbb{R}^{\mathrm{n}}, \mathrm{C}^{\infty}\left(\partial \mathrm{M}, \mathbb{R}^{\mathrm{n}}\right)\right)$ uniquely determined up to maps in $C^{\infty}\left(E\left(M, \mathbb{R}^{n}\right), \mathbb{R}^{n}\right)$, such that

$$
F(j)(l)=\int_{\partial M}<<\Delta(j) h(d j), l>\mu(j), \forall j \in O, \forall l \in C^{\infty}\left(\partial M, \mathbb{R}^{n}\right)
$$

(iv.) There is a unique smooth map $\varphi \in C^{\infty}\left(O, C^{\infty}\left(\partial M, \mathbb{R}^{n}\right)\right)$, such that

$$
\mathrm{F} \partial(\mathrm{j})(\mathrm{l})={ }_{\partial \mathrm{M}} \int\left\langle\varphi(\mathrm{dj}), \mathrm{l}>\mu(\mathrm{j}), \quad \forall \mathrm{j} \in O, \forall \mathrm{l} \in \mathrm{C}^{\infty}\left(\partial \mathrm{M}, \mathbb{R}^{\mathrm{n}}\right),\right.
$$

and which satisfies

$$
\int_{\mathrm{M}} \int\left\langle\varphi(\mathrm{dj}), \mathrm{z}>\mu(\mathrm{j})=0, \quad \forall \mathrm{j} \in \mathrm{O}, \forall \mathrm{z} \in \mathbb{R}^{\mathrm{n}}\right.
$$

For an explicit formulation of $\Delta(\mathrm{j})$ in terms of the coefficients of dj we refer to $[\mathrm{Bi}, \mathrm{Sc}, \mathrm{So}]$.

\section{Remark :}

The remark at the end of section 4 translates accordingly to the situation studied in this section.

* Next we will investigate a boundary material implemented to a body formed by a deformable medium. 


\section{- 6. The interplay between constitutive laws of boundary and body}

- The deformable media forming the inside of the body and the boundary respectively may differ and each separate material hence has to be described on one hand by different constitutive laws. This we have done in the previous sections. On the other hand these materials together form one body and should be describable by only one constitutive law holding for the whole body. Since constitutive laws behave additively, the comparision between the two procedures allows us to decode the influence of the whole body to the constitutive properties of the boundary material.

Let the constitutive law of the deformable medium forming the whole body be called by $\mathrm{F}$ again.

According to theorem $4.2, \mathrm{~F}$ is determined by a smooth constitutive map

$$
\mathfrak{H}: \mathrm{E}\left(\mathrm{M}, \mathbb{R}^{\mathrm{n}}\right) /_{\mathbb{R}^{n}} \rightarrow \mathrm{C}^{\infty}\left(\mathrm{M}, \mathbb{R}^{\mathrm{n}}\right)
$$

: The following theorem exhibits its influence to the constitutive entities of the material forming the boundary of the body. We will indicate next the methods to : prove it.

The map $\mathfrak{H}$ yields according to theorem 4.2 force densities

$$
\Phi: E\left(M, \mathbb{R}^{n}\right) / \mathbb{R}^{n} \rightarrow C^{\infty}\left(M, \mathbb{R}^{n}\right)
$$

and

$$
\varphi: E\left(M, \mathbb{R}^{n}\right) / \mathbb{R}^{n} \rightarrow C^{\infty}\left(\partial M, \mathbb{R}^{n}\right)
$$

The force density acting on $\partial \mathrm{M}$, is defined by

$$
\varphi(\mathrm{d} J)=\mathrm{d} \mathfrak{S}(\mathrm{d} J)(\mathrm{N}), \quad \forall \mathrm{d} J \in E\left(M, \mathbb{R}^{\mathrm{n}}\right) / \mathbb{R}^{\mathrm{n}}
$$

Having the integrability condition (5.14) of $\Delta(J)$ in mind, we split this force density $\varphi$ into

$$
\varphi(\mathrm{dJ})=\varphi_{\mathbb{R}^{n}}(\mathrm{dJ})+\psi(\mathrm{dJ}), \quad \forall \mathrm{dJ} \in \mathrm{E}\left(\mathrm{M}, \mathbb{R}^{\mathrm{n}}\right) /_{\mathbb{R}^{\mathrm{n}}}
$$

where $\varphi_{\mathbb{R}^{n}}(\mathrm{dJ})$ is characterized for each $\mathrm{dJ} \in \mathrm{E}\left(\mathrm{M}, \mathbb{R}^{\mathrm{n}}\right) /_{\mathbb{R}^{n}}$ by the equation 
(6.5)

$$
\partial\left\langle\mathrm{M}^{\mathrm{n}}(\mathrm{dJ}), \mathrm{z}>\mathrm{i}_{\mathrm{N}} \mu(J)=0, \quad \forall \mathrm{z} \in \mathbb{R}^{\mathrm{n}}\right.
$$

and where

$$
\psi: \mathrm{E}\left(\mathrm{M}, \mathbb{R}^{\mathrm{n}}\right) \mathbb{\mathbb { R }}^{\mathrm{n}} \rightarrow \mathbb{R}^{\mathrm{n}}
$$

is a smooth map, which makes (6.4) to hold.

According to theorem 5.1 the condition (6.5) allows us to choose some map

$$
\mathfrak{h}: E\left(M, \mathbb{R}^{\mathrm{n}}\right) / \mathbb{R}^{\mathrm{n}} \rightarrow \mathrm{C}^{\infty}\left(\partial \mathrm{M}, \mathbb{R}^{\mathrm{n}}\right)
$$

such that for all $d J \in E\left(M, \mathbb{R}^{n}\right) / \mathbb{R}^{n}$ the equation

$$
\varphi_{\mathbb{R}} \mathrm{n}(\mathrm{dJ})=\Delta(\mathrm{J} \mid \partial \mathrm{M}) \mathfrak{h}(\mathrm{dJ})
$$

holds true.

\section{: Theorem 6.1:}

Any smoothly deformable medium is characterized by a constitutive map

$$
\mathfrak{H}: E\left(M, \mathbb{R}^{n}\right) / \mathbb{R}^{n} \rightarrow C^{\infty}\left(M, \mathbb{R}^{n}\right)
$$

determining itself two smooth maps

$$
\mathfrak{h}: \mathrm{E}\left(\mathrm{M}, \mathbb{R}^{\mathrm{n}}\right){\mathbb{\mathbb { R } ^ { n }}}^{\mathrm{n}} \rightarrow \mathrm{C}^{\infty}\left(\partial \mathrm{M}, \mathbb{R}^{\mathrm{n}}\right)
$$

and

$$
\psi: \mathrm{E}\left(\mathrm{M}, \mathbb{R}^{\mathrm{n}}\right) \rightarrow \mathbb{R}^{\mathrm{n}}
$$

which are linked to $\mathfrak{H}$ by the boundary condition

$$
\mathrm{d} \mathfrak{H}(\mathrm{dJ})(\mathrm{N})=\Delta(\mathrm{J} \mid \partial \mathrm{M}) \mathfrak{h}(\mathrm{dJ})+\psi(\mathrm{dJ}) .
$$

$\mathfrak{h}$ is unique up to $\mathbb{R}^{\mathrm{n}}$-valued smooth maps of $\mathrm{E}\left(\mathrm{M}, \mathbb{R}^{\mathrm{n}}\right)$ and $\psi$ is unique. Moreover $\mathfrak{H}$ satisfies the integrability conditions 


$$
0=\int_{M} \int<\Delta(J) \mathfrak{H}(d J), \mathrm{z}>\mu(J)+{ }_{\partial M} \int<\mathrm{d} \mathfrak{H}(\mathrm{d} J)(\mathrm{N}), \mathrm{z}>\mathrm{i}_{N} \mu(J),
$$

for each $J \in E\left(M, \mathbb{R}^{n}\right)$ and each $z \in \mathbb{R}^{n}$. Equation (6.11) equivalently formulated reads as

$$
\begin{aligned}
0= & \int<\Delta(\mathrm{J}) \mathfrak{H}(\mathrm{dJ}), \mathrm{z}>\mu(\mathrm{J}) \\
& +{ }_{\partial \mathrm{M}^{\int}} \int<\psi(\mathrm{dJ}), \mathrm{z}>\mathrm{i}_{\mathrm{N}} \mu(\mathrm{J}),
\end{aligned}
$$

a boundary condition holding for $\mathfrak{H}$ and $\psi$ together. The constitutive law on $\mathrm{E}\left(\mathrm{M}, \mathbb{R}^{\mathrm{n}}\right)$ describing the constitutive properties of the materials forming body together with its boundary is thus given via the formula

$$
\begin{aligned}
& \mathrm{F}(\mathrm{J})(\mathrm{L})={ }_{\mathrm{M}}^{\int<\Delta(J) \mathfrak{H}(J), \mathrm{L}>\mu(\mathrm{J})} \\
& \left.+{ }_{\partial \mathrm{M}} \int<\Delta(\mathrm{J} \mid \partial \mathrm{M}) \mathrm{h}(\mathrm{J} \mid \partial \mathrm{M})+\psi(\mathrm{d} J)\right), \mathrm{L}>\mathrm{i}_{\mathrm{N}} \mu(\mathrm{J}), \\
& \forall J \in E\left(M, \mathbb{R}^{n}\right), \forall L \in C^{\infty}\left(M, \mathbb{R}^{n}\right) .
\end{aligned}
$$

The work of any distortion $l \in C^{\infty}\left(\partial M, \mathbb{R}^{n}\right)$ of the deformable material forming the : boundary detached from the body is for any $J \in E\left(M, \mathbb{R}^{n}\right)$ given by

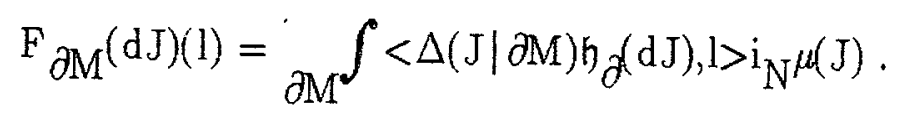

for some constitutive map $\mathfrak{h}_{\partial} \in \mathrm{C}^{\infty}\left(\mathrm{O} \partial / \mathbb{R}^{\mathrm{n}, \mathrm{C}^{\infty}}\left(\partial \mathrm{M}, \mathbb{R}^{\mathrm{n}}\right)\right)$.

The constitutive properties of the deformable medium of the boundary detached from the body, which is given by a smooth map $\mathfrak{h}_{\partial} \in \mathrm{C}^{\infty}\left(\mathrm{O}_{\partial} / \mathbb{R}^{\mathrm{n}}, \mathrm{C}^{\infty}\left(\partial \mathrm{M}, \mathbb{R}^{\mathrm{n}}\right)\right)$. Hence $\mathfrak{h}-\mathfrak{h} \partial$ and $\psi$ describe how the constitutive properties of the material forming the boundary of the body is affected by the fact that this material is incorporated into the material forming the whole body. 


\section{Simple examples}

In this section we will study well known one forms on $E\left(M, \mathbb{R}^{n}\right)$ in the light of our

- formalism developed above. We do so by looking at particularly simple constitutive maps.

(i.) In our first example we specify a constitutive map by $\mathfrak{S}(\mathrm{dJ})=\mathrm{J}$ for all $J \in E\left(M, \mathbb{R}^{n}\right)$. The following calculation is easily verified :

$$
\begin{aligned}
& \mathrm{M}^{\mathrm{J} \mathrm{dJ} \cdot \mathrm{dL} \mu(\mathrm{J})} \\
& =\int<\Delta(J) J, L>\mu(J)+{ }_{\partial M} \int<d J(N), l>i_{N} \mu(J) \\
& =\int_{M} t_{\Gamma} A(d L, d J) \mu(J) \\
& =\int_{M} \operatorname{tr} \nabla(J) X(L, J) \mu(J) \\
& =\int_{\mathrm{M}^{2}} \operatorname{div}_{\mathrm{J}} \mathrm{X}(\mathrm{L}, \mathrm{J}) \mu(\mathrm{J}) \\
& ={ }_{\partial M^{2}} \int<N(j), L>\mu(j) \\
& =\mathrm{D}\left(\int_{\mathrm{M}} \int \mu(\mathrm{J})\right)(\mathrm{L}) \text {, }
\end{aligned}
$$

for all $J \in E\left(M, \mathbb{R}^{n}\right)$ and for all $L \in C^{\infty}\left(M, \mathbb{R}^{n}\right)$.

Introducing the volume function

$$
\mathfrak{V}: E\left(M, \mathbb{R}^{n}\right) \rightarrow \mathbb{R}
$$

assigning to any $J \in E\left(M, \mathbb{R}^{n}\right)$ the volume

$$
\mathfrak{V}(J)=\int_{M} \mu(J)
$$

we have

$$
\mathrm{D} \mathfrak{V}(J)(\mathrm{L})={ }_{M}^{\int \mathrm{d} J \cdot \mathrm{dL} \mu(J)}
$$

with $\mathrm{N}(\mathrm{j}):=\mathrm{TJN}$ together with the following notions $\mathrm{l}:=\mathrm{L} \mid \partial \mathrm{M}$ and $\mathrm{j}:=\mathrm{J} \mid \partial \mathrm{M}$.

The above calculation shows

and

$$
\Phi(\mathrm{dJ})=\Delta(J) J=0, \quad \forall J \in E\left(M, \mathbb{R}^{\mathbf{n}}\right)
$$

$$
\varphi(\mathrm{dJ})=\mathrm{N}(\mathrm{j}), \quad \forall \mathrm{J} \in \mathrm{E}\left(\mathrm{M}, \mathbb{R}^{\mathrm{n}}\right)
$$


Clearly $\mathrm{l}=\mathrm{z}$ with $\mathrm{z} \in \mathbb{R}^{\mathrm{n}}$ implies

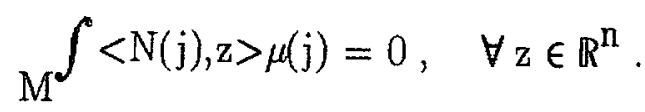

With the notations of the previous section this shows that in this example

$$
\varphi=\varphi_{\mathbb{R}^{n}}
$$

The map $\mathfrak{h}$ determined by $\mathfrak{H}$ in this case is thus given by

$$
\mathrm{N}(\mathrm{J})=\Delta(\mathrm{j}) \mathrm{h}(\mathrm{dJ}), \quad \forall \mathrm{J} \in \mathrm{E}\left(\mathrm{M}, \mathbb{R}^{\mathrm{n}}\right) \text { and } \mathrm{j}:=\mathrm{J} \mid \partial \mathrm{M}
$$

(ii.) Next let us turn our attention to $\mathfrak{h}_{\partial}$ on $E\left(\partial M, \mathbb{R}^{n}\right)$, given by $\mathfrak{h} \not(d j)=j$, for all $j \in E\left(\partial M, \mathbb{R}^{n}\right)$. One easily verifies the following set of equations

$$
\begin{aligned}
& { }_{\partial \mathrm{M}} \int<\Delta(\mathrm{j}) \mathrm{j}, \mathrm{l}>\mu(\mathrm{j})={ }_{\partial \mathrm{M}} \int \mathrm{dj} \cdot \mathrm{dl} \mu(\mathrm{j}) \\
& ={ }_{\partial \mathrm{M}} \int\left(\operatorname{div}_{\mathrm{j}} \mathrm{X}(\mathrm{l}, \mathrm{j})+\theta(\mathrm{l}, \mathrm{j}) \cdot \mathrm{H}(\mathrm{j})\right) \mu(\mathrm{j}) \\
& =D\left({ }_{\partial \mathrm{M}} \int \mu(\mathrm{j})\right)(\mathrm{l}) \text {. }
\end{aligned}
$$

Defining the area function

$$
\mathfrak{A}: \mathrm{E}\left(\partial \mathrm{M}, \mathbb{R}^{\mathrm{n}}\right) \rightarrow \mathbb{R}
$$

sending any $j \in E\left(\partial M, \mathbb{R}^{n}\right)$ into

$$
\mathfrak{A}(\mathrm{j})={ }_{\partial \mathrm{M}} \int \mu(\mathrm{j})
$$

we have

$$
\mathrm{D} \mathfrak{A}(\mathrm{j})(\mathrm{l})={ }_{\partial \mathrm{M}} \int \mathrm{dj} \cdot \mathrm{dl} \mu(\mathrm{j})
$$

for all variables of $\mathrm{D} \mathfrak{A}$. The constitutive map $\mathfrak{h}_{\partial}$ determines a map $\mathfrak{H}_{\partial}$ given by

$$
0=\Delta(J) \mathfrak{H} \not(\mathrm{dJ}), \quad \forall J \in \mathrm{E}\left(\mathrm{M}, \mathbb{R}^{\mathrm{n}}\right)
$$

together with 


$$
d \mathfrak{H} \partial(d J)(N)=\Delta(j) j=H(j) \cdot N(j)
$$

for each $J \in E\left(M, \mathbb{R}^{n}\right)$ and $j:=J \mid \partial M$. The function $H(j)$ is the mean curvature, that is the trace of $W(j)$.

(iii.) Next let us consider quite another influence of the boundary by looking at the

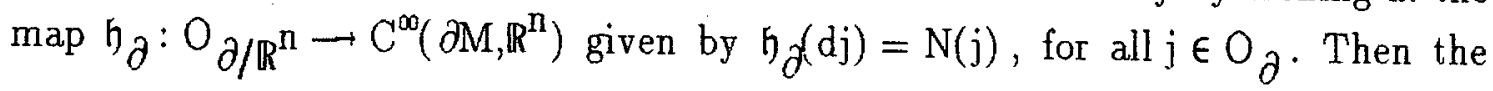
formula

$$
\begin{aligned}
& \Delta(\mathrm{j}) \mathrm{N}(\mathrm{j})=\delta \mathrm{dN}(\mathrm{J}) \\
& =\operatorname{ddj} W(\mathrm{j}) \\
& =-\operatorname{djg}_{\operatorname{grad}_{j}} \mathrm{H}(\mathrm{j})+\left(\operatorname{tr} W(\mathrm{j})^{2}\right) \cdot N(\mathrm{j})
\end{aligned}
$$

holds for any $j \in O_{\partial}$. Let us point out that $\Delta(j) N(j) \neq 0$ even if $j(\partial M) \subset \mathbb{R}^{n}$ is minimal, that is to say even if $\mathrm{H}(\mathrm{j})=$ const.

- In the special case of $\operatorname{dim} \partial \mathrm{M}=2$ a topological constant, the Euler characteristic ; $N(\partial M)$, enters the constitutive law $F$ determined by $N(j)$ for each $j \in O_{\partial}$. It is hidden in the formula

$$
\begin{aligned}
F(j)(N(j))= & \int\langle\Delta(j) N(j), N(j)>\mu(j) \\
& ={ }_{\partial M} \int \operatorname{tr} W(j)^{2} \mu(j) .
\end{aligned}
$$

This is seen by using the Cayley Hamilton theorem (cf.[Gr]) and the Gauss Bonnet theorem (cf.[G,H,V]), applied to the right hand side of equation (7.16), which yields

$$
\mathrm{F}(\mathrm{j})(\mathrm{N}(\mathrm{j}))=-4 \pi \chi(\partial \mathrm{M})+{ }_{\partial \mathrm{M}} \int \mathrm{H}(\mathrm{j})^{2} \mu(\mathrm{j})
$$

\section{A general decomposition of constitutive laws}

In this section we will exhibit a decomposition of the constitutive map $\mathfrak{H}$ based on the examples of the previous section. 
We will show that $D \mathfrak{V}$ and $R^{*} D \mathfrak{A}$ multiplied with appropriate $\mathbb{R}$-valued maps are - all part of any constitutive law $\mathrm{F}$ defined on $\mathrm{E}\left(\mathrm{M}, \mathbb{R}^{\mathrm{n}}\right)$.

- To get the full decomposition of a given constitutive law we broaden our scope a little and introduce first of all the Hilbert space $A_{i}$ consisting of all maps $\gamma_{1}, \gamma_{2}: \mathrm{TM} \rightarrow \mathbb{R}$ linear on the fibres of TM for which the right hand side of

$$
G_{\mathbb{R}^{n}}^{\partial}(\mathrm{dj})\left(\gamma_{1}, \gamma_{2}\right):={ }_{\partial M^{2}}^{\int \gamma_{1} \cdot \gamma_{2} \mu(j)}
$$

exists. Clearly $\mathrm{dh}_{\mathrm{N}}(\mathrm{dJ}), \mathrm{dj}$ and $\mathrm{dN}(\mathrm{j})$ all belong to $\mathrm{A}_{\mathrm{j}}$ and are generically linearly independent. The set $O_{3}$ of all $J \in E\left(M, \mathbb{R}^{n}\right)$ for which these three differentials are linearly independent form a dense open set. Let $j:=J \mid \partial M$. In special case $j(\partial M)$ is a (n-1)-sphere in $\mathbb{R}^{n}$ however, $N(j)$ is a real multiple, $r$ say, of $j$ and $\mathfrak{h}_{N}(d J)$ is hence $\frac{r}{(n-1)} \cdot j$. In the case of linear independence the three above mentioned - differentials are in general (with respect to $G_{\mathbb{R}} n\left(d_{j}\right)$ ) not orthogonal to each other, however. We might orthogonalize them by using the method of Schmidt. For each $J \in O_{3}$ we then split the differential of $\mathfrak{h}(\mathrm{dJ})$ into components along span of the

: three mentioned differentials and a component perpendicular to them.

Next we extend all maps $\mathfrak{h}_{\mathrm{N}}(\mathrm{dJ}), \mathrm{j}$ and $\mathrm{N}(\mathrm{j})$ to all of $M$ in the following way :

Given $f \in C_{j}^{\infty}\left(\partial M, \mathbb{R}^{n}\right)$ we solve the following Višik problem (cf.[Hö]) :

$$
\begin{gathered}
\Delta(J) f_{M}=0 \\
\operatorname{df}_{M}(N)-\Delta(j) f=0
\end{gathered}
$$

with $f_{M} \subset C^{\infty}\left(M, \mathbb{R}^{n}\right)$ and $J \in E\left(M, \mathbb{R}^{n}\right)$ and where $j:=J \mid \partial M$. All the splittings and extensions done to construct $j_{M}$ and $N(j)_{M}$ depend smoothly on $j \in E\left(\partial M, \mathbb{R}^{n}\right)$. Let moreover $\mathfrak{h}_{\mathrm{N}}$ be given by

$$
\left(\mathfrak{h}_{N}(\mathrm{dJ})\right)_{M}=J, \quad \forall J \in E\left(M, \mathbb{R}^{n}\right)
$$

The above mentioned decomposition of $\mathfrak{H}$ is then described in the following - theorem : 


\section{Theorem 8.1 :}

Let $F$ be a constitutive law on $E\left(M, \mathbb{R}^{n}\right)$ determined by

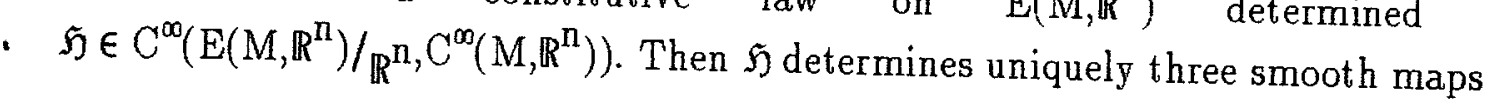

$$
a_{1}, a_{2}, a_{3}: O_{3} \subset E\left(M, \mathbb{R}^{n}\right) / \mathbb{R}^{n} \rightarrow \mathbb{R}
$$

and two smooth maps

$$
\mathfrak{h}, \mathfrak{h}^{2}: \dot{O}_{3}^{\cdot} \subset \mathrm{E}\left(\mathrm{M}, \mathbb{R}^{\mathrm{n}}\right) /_{\mathbb{R}^{n}} \rightarrow \mathrm{C}^{\infty}\left(\partial \mathrm{M}, \mathbb{R}^{\mathrm{n}}\right)
$$

such that the following splitting holds for any $d J \in O_{3} \subset E\left(M, \mathbb{R}^{n}\right)$

$$
\mathfrak{h}(d J)=a_{1}(d J) \cdot \mathfrak{h}_{N}(d J)+a_{2}(d J) j+a_{3}(d J) \cdot N(j)+\mathfrak{h}_{2}(d J)
$$

with $\mathrm{j}:=\mathrm{j} / \partial \mathrm{M}$. The differential $d h_{2}(\mathrm{dJ})$ is orthogonal with respect to $G(\mathrm{dj})_{\mathbb{R}^{n}}^{\partial}$ to the span of $\mathrm{dh}_{\mathrm{N}}(\mathrm{d} J)$, dj and $\mathrm{dN}(\mathrm{j})$.

;

The map $\mathfrak{H}(\mathrm{d} J)$ decomposes for each $\mathrm{J} \in \mathrm{O}_{3}$ accordingly into

$$
\mathfrak{H}(d J)=a_{1}(d J) \cdot J+a_{2}(d J) \cdot J_{M}+a_{3}(d J) \cdot N_{M}(d J)+\mathfrak{H}_{2}(d J)
$$

with $\mathrm{j}:=\mathrm{J} \mid \partial \mathrm{M}$ and where $\mathfrak{H}_{2}(\mathrm{~d} J)$ is such that $(8.10)$ holds.

\section{The rotational symmetry}

Finally we investigate the effect of the $S O(n)$, the symmetry group of the oriented Euclidean vector space $\left(\mathbb{R}^{n},<,>, \underline{\Delta}\right)$ to a constitutive law. In particular let us - characterize those which are invariant under $S O(n)$.

- As mentioned in the first section we have the operation

$$
\mathfrak{s}: \mathrm{SO}(\mathbf{n}) \times \mathrm{E}\left(\mathrm{M}, \mathbb{R}^{\mathrm{n}}\right) \longrightarrow \mathbb{R}^{\mathrm{n}}
$$


sending any $g \in S O(n)$ and any $J \in E\left(M, \mathbb{R}^{n}\right)$ into

$$
\mathfrak{s}(\mathrm{g}, J)=g \circ J
$$

Let $\mathfrak{s}_{\mathrm{g}}(\mathrm{J}):=\mathrm{g} \circ \mathrm{J}$ for each pair $\mathrm{g} \in \mathrm{SO}(\mathrm{n})$ and each $\mathrm{J} \in \mathrm{E}\left(\mathrm{M}, \mathbb{R}^{\mathrm{n}}\right)$.

The induced operation $\mathfrak{s}_{T}$ on $T E\left(M, \mathbb{R}^{n}\right)$ is therefore determined by

$$
\begin{gathered}
\mathfrak{s}_{\mathrm{T}}: S O(\mathrm{n}) \times \mathrm{E}\left(\mathrm{M}, \mathbb{R}^{\mathrm{n}}\right) \times C^{\infty}\left(M, \mathbb{R}^{\mathbf{n}}\right) \rightarrow \mathrm{E}\left(\mathrm{M}, \mathbb{R}^{\mathrm{n}}\right) \times C^{\infty}\left(\mathrm{M}, \mathbb{R}^{\mathrm{n}}\right) \\
{ }_{\mathrm{s}} \mathrm{T}(\mathrm{g},(J, L))=(\mathrm{g} \circ \mathrm{J}, \mathrm{g} \circ \mathrm{L})
\end{gathered}
$$

for all $g \in S O(n)$, for all $J \in E\left(M, \mathbb{R}^{n}\right)$ and for all $L \in C^{\infty}\left(M, \mathbb{R}^{n}\right)$.

Invariance under $S O(n)$ of a given constitutive law $F$ with $\mathfrak{H}$ means that the following equation

$$
F(g \circ J)(g \circ L)=F(J)(L)
$$

holds for all the variables of $\mathrm{F}$ and for any $\mathrm{g} \in \mathrm{SO}(\mathrm{n})$. Thus the constitutive map
satisfies

$$
{ }_{M} \int \mathrm{d} \mathfrak{H}(\operatorname{god} J) \cdot d(g \circ L) \mu(g \circ J)={ }_{M} \int d \mathfrak{H}(d J) \cdot d L \mu(J)
$$

or reformulated

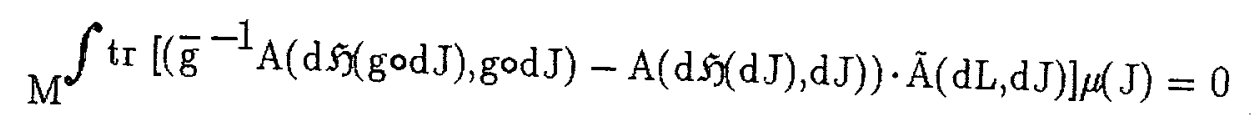

with $\overline{\mathrm{g}}=(\mathrm{dJ})^{-1} \circ \mathrm{g}$. Thus $\overline{\mathrm{g}} \in \mathrm{SO}\left(\mathrm{T}_{\mathrm{p}} \mathrm{M}\right)$, the special orthogonal group of $\mathrm{T}_{\mathrm{p}} \mathrm{M}$ for each $p \in M$. The volume $\mu$ is invariant under the action $\mathfrak{s}_{T}$. Hence

$$
\mathrm{M}^{\int \mathrm{d}\left(\mathrm{g}^{-1} \circ \mathfrak{H}(\operatorname{g\circ d} J)-\mathfrak{H}(\mathrm{d} J)\right) \cdot \mathrm{dL} \mu(J)=0,}
$$

"holds for all $g \in S O(n)$, for all $J \in E\left(M, \mathbb{R}^{n}\right)$ and for all $L \in C^{\infty}\left(M, \mathbb{R}^{n}\right)$. From the last equation and from the general procedure of representing differentials via embeddings we read off 


\section{Proposition 9.1:}

- Given a constitutive law $F$ with constitutive map $\mathfrak{H} \in \mathrm{C}^{\infty}\left(\mathrm{E}\left(\mathrm{M}, \mathbb{R}^{\mathrm{n}}\right), \mathrm{C}^{\infty}\left(\mathrm{M}, \mathbb{R}^{\mathrm{n}}\right)\right)$ then $F$ is invariant under $S O(n)$ iff

$$
\mathrm{g}^{-1} \circ \mathrm{d} \mathfrak{H}(\operatorname{god} J)=\mathrm{d} \mathfrak{H}(\mathrm{dJ}), \quad \forall \mathrm{G} \in \mathrm{SO}(\mathrm{n})
$$

The validity of this equation is equivalent with the following identity

$$
\mathrm{A}(\mathrm{d} \mathfrak{S}(\operatorname{god} J), \operatorname{g\circ d} J)(p)=A(d \mathfrak{S}(d J), d J)(p)
$$

holding for all $g \in S O(n)$, for all $p \in M$ and for all $J \in E\left(M, \mathbb{R}^{n}\right)$. Moreover the stress tensor $T(J)$ determined by $\mathfrak{H}$ is invariant under $S O(n)$ for any $J \in E\left(M, \mathbb{R}^{n}\right)$.

Let $\mathrm{F}$ be a $S O(\mathrm{n})$-invariant constitutive law. (9.5) implies that for each $\mathrm{c} \in \mathrm{so}(\mathrm{n})$

$$
\operatorname{cod} \mathfrak{S}(d J)=d D \mathfrak{H}(d J)(\operatorname{cod} J)
$$

: with $D$ the derivative on $E\left(M, \mathbb{R}^{n}\right) \mathbb{R}_{\mathbb{R}^{n}}$. Here so(n) is the Lie-algebra of $S O(n)$. Based on (9.7) it is a matter of routine to show that in case $\mathfrak{H}$ is invariant under $S O(n)$ then $c d \mathfrak{H}(d J)=0$ for any $c \in \operatorname{so}(n)$ and any $J \in E\left(M, \mathbb{R}^{n}\right)$. In case of $\operatorname{dim} M=3$ this means that the $S O(n)$-invariance of $\mathfrak{S}(J)$ yields $\mathfrak{S}(d J)=$ const. and in turn that $\mathrm{F}=0$.

Splitting $A(d \mathfrak{H}(d J), d J)$ with respect to $m(J)$ into symmetric and skew-symmetric part and doing the same for $\mathrm{A}(\mathrm{dD} \mathcal{S}(\mathrm{dJ})(\operatorname{cod} J), \mathrm{dJ})$ and using (9.7) yields

$$
\begin{array}{r}
\bar{c} \circ B(d \mathfrak{H}(d J), d J)(p)+\bar{c} \circ C(d \mathfrak{H}(d J), d J)(p) \\
=B(d D \mathfrak{H}(d J)(\operatorname{cod} J), d J)(p)+C(d D \mathfrak{H}(d J)(\operatorname{cod} J), d J)(p)
\end{array}
$$

with $\bar{c} \in$ so( $\left.T_{p} M\right)$ for each fixed $p \in M$. Here so $\left(T_{p} M\right)$ denotes the Lie Algebra of $S O\left(T_{p} M\right)$. If tr $A(d D F(d J)(\operatorname{cod} J), d J)=0$ then (9.8) yields

$$
\operatorname{tr} \bar{c} \circ C(d \mathfrak{H}(d J), d J)(p)=0
$$$$
\forall \mathrm{c} \in \mathrm{so}(\mathrm{n}), \forall \mathrm{p} \in \mathrm{M}
$$$$
\text { and } \forall J \in E\left(M, \mathbb{R}^{\mathrm{n}}\right) \text {. }
$$

and vice versa. This implies the following theorem : 


\section{Theorem 9.2 :}

- For any $S O(n)$-invariant constitutive law with (smooth) constitutive map $\mathfrak{H}$ the following are equivalent:

$$
\begin{gathered}
C(\mathrm{~d} S(\mathrm{~d} J), \mathrm{d} J)=0, \quad \forall J \in E\left(M, \mathbb{R}^{\mathrm{n}}\right) \\
\operatorname{tr} \mathrm{A}(\mathrm{dD} \mathfrak{S}(\mathrm{d} J)(\operatorname{cod} J), \mathrm{d} J)=0 \quad \forall J \in E\left(M, \mathbb{R}^{\mathbf{n}}\right)
\end{gathered}
$$

(9.12) The stress tensor $T(J)$ associated with $\mathfrak{H}$ is symmetric for each configuration $\mathrm{J}$.

Let us illustrate (9.11) somewhat. In doing so 'we proceed similar as in section 8 and take the components of $d \mathfrak{H}(g \cdot d J)$ and $d \mathfrak{H}(d J)$ along $d J$, this is to say we have the splitting

$$
\mathrm{d} \mathfrak{H}(\mathrm{g} \cdot \mathrm{d} J)=\hat{\Pi}(\mathrm{g} \cdot \mathrm{d} J) \cdot \mathrm{d} J+\hat{d} \hat{\mathfrak{H}}_{1}(g \cdot d J)
$$

and

(9.14)

$$
\mathrm{d} \mathfrak{H}(\mathrm{d} J)=\Pi(\mathrm{dJ}) \cdot \mathrm{d} J+\mathrm{d} \mathfrak{H}_{1}(\mathrm{~d} J)
$$

where both $d \hat{H}_{1}(d J)$ and $d \mathfrak{H}_{1}(d J)$ are orthogonal to $d J$ with respect to $G_{\mathbb{R}} n(d J)$. Both $\Pi$ and $\hat{\Pi}$ can be regarded as internal pressures. All the maps in (9.13) and (9.14) are smooth. We therefore find

$$
\begin{aligned}
\mathrm{M}^{\int \operatorname{tr}} & A(\mathrm{~d} \mathfrak{H}(\mathrm{g} \cdot \mathrm{d} J), \mathrm{d} J) \mu(J)=n \cdot \hat{\Pi}(\mathrm{g} \cdot \mathrm{d} J) \cdot \mathfrak{N}(J) \\
= & n \cdot \Pi(\mathrm{dJ}) \cdot \mathfrak{V}(J) \cdot \operatorname{tr} \mathrm{g}+\int_{M} \operatorname{tr} \mathrm{A}\left(\mathrm{g} \cdot \mathrm{d} \hat{\mathfrak{H}}_{1}(\mathrm{dJ}), \mathrm{d} J\right) \mu(J)
\end{aligned}
$$

and in turn

$$
\mathrm{M}^{\int \operatorname{tr} A(d D \mathfrak{H}(d J)(c \cdot d J), d J)=n \cdot \mathfrak{V}(J) \cdot D \hat{\Pi}(d J)(c \cdot d J)}
$$


Hence (9.11) requires

(9.17)

$$
\mathrm{D} \hat{\Pi}(\mathrm{dJ})(\mathrm{c} \cdot \mathrm{dJ})=0, \quad \forall J \in E\left(M, \mathbb{R}^{\mathrm{n}}\right) \text { and } \forall \mathrm{c} \in \mathrm{so}(\mathrm{n}) \text {, }
$$

showing that $\hat{\Pi}$ is up to the first order invariant under $S O(n)$. 
References:

[A,M,R] R.Abraham, J.E.Marsden, T.Ratiu : Manifolds, Tensor Analysis, and Applications,

Addison Wesley, Global Analysis, 1983

[Bi] E.Binz : On the Notion of the Stress Tensor Associated with $\mathrm{R}^{\mathrm{n}}$-invariant Constitutive Laws Admitting Integral Representations, Reports on Mathematical Physics, Vol.87, (1989), p.49-57

[Bi,Sc,So] E.Binz, G.Schwarz, D.Socolescu : On a Global Differential Geometric Description of the Motions of Deformable Media, to appear in Infinite Dimensional Manifolds, Groups, and Algebras, Vol. II, Ed.H.D.Doebner, J.Hennig, 1990

[Bi,Sn,Fi] E.Binz, J.Śniatycki, H.Fischer: Geometry of Classical Fields, Mathematics Studies 154, North-Holland Verlag, Amsterdam, 1988

[Fr, Kr] A.Frölicher, A.Kriegl : Linear Spaces and Differentiation Theory, John Wiley, Chichester, England, 1988

[G,H,V] W.Greub, S.Halperin, J.Vanstone : Connections, Curvature and Cohomology, I, II, Acad. Press, New York, 1972-73

[Gr] W.Greub : Lineare Algebra I, Graduate Texts in Mathematics, Vol.23, Springer Verlag, Berlin, Heidelberg, New York, 1981

[E,S] M.Epstein, R.Segev : Differentiable Manifolds and the Principle of Virtual Work in Continuum Mechanics, Journal of Mathematical Physics, No.5, Vol.21, (1980)

[He] E.Hellinger : Die allgemeinen Ansätze der Mechanik der Kontinua, Enzykl. Math. Wiss. 4/4, 1914

[Hi]

M.W.Hirsch : Differential Topology, Springer GTM, Berlin, 1976

[Ḧ̈]

L.Hörmander : Linear Partial Differential Operations, Grundlehren der mathematischen Wissenschaften, Vol.116, Springer Verlag, Berlin, Heidelberg, New York, 1976 
-

F.John : Partial Differential Equations,

Applied Mathematical Science, Vol.1, (1978)

[L,L] L.D.Landau, E.M.Lifschitz : Lehrbuch der theoretischen Physik, Vol. VII, Elastizitätstheorie, 4. Auflage,

Akademie Verlag, Berlin, 1975

[Mat] Y.Matsushima : Vector Bundle Valued Canonic Forms, Osaka Journal of Mathematics, Vol.8, (1971) 